\title{
CONTROL OF THE CONTINUITY EQUATION WITH A NON LOCAL FLOW
}

\author{
Rinaldo M. Colombo ${ }^{1}$, Michael Herty ${ }^{2}$ And Magali Mercier ${ }^{3}$
}

\begin{abstract}
This paper focuses on the analytical properties of the solutions to the continuity equation with non local flow. Our driving examples are a supply chain model and an equation for the description of pedestrian flows. To this aim, we prove the well posedness of weak entropy solutions in a class of equations comprising these models. Then, under further regularity conditions, we prove the differentiability of solutions with respect to the initial datum and characterize this derivative. A necessary condition for the optimality of suitable integral functionals then follows.
\end{abstract}

Mathematics Subject Classification. 35L65, 49K20, 93C20.

Received February 13, 2009. Revised September 30, 2009.

Published online March 24, 2010.

\section{INTRODUCTION}

We consider the scalar continuity equation in $N$ space dimensions

$$
\left\{\begin{array}{lc}
\partial_{t} \rho+\operatorname{div}(\rho V(\rho))=0 & (t, x) \in \mathbb{R}_{+} \times \mathbb{R}^{N} \\
\rho(0, x)=\rho_{0}(x) & x \in \mathbb{R}^{N}
\end{array}\right.
$$

with a non local speed function $V$. This kind of equations appears in numerous examples, a first one being the supply chain model introduced in $[3,4]$, where $V(\rho)=v\left(\int_{0}^{1} \rho(x) \mathrm{d} x\right)$, see Section 3. Another example comes from pedestrian traffic, in which a reasonable model can be based on (1.1) with the functional $V(\rho)=$ $v(\rho * \eta) \vec{v}(x)$, see Section 4. Throughout, our assumptions are modeled on these examples. Other analytically similar situations are found in [5], where a kinetic model for pedestrians is presented, and in [23], which concerns the Keller-Segel model.

In the following we postulate assumptions on the function $V$ which are satisfied in the cases of the supply chain model and of the pedestrian model, but not for general functions. In particular, we essentially require below that $V$ is a non local function, see (2.1).

The first question we address is that of the well posedness of (1.1). Indeed, we show in Theorem 2.2 that (1.1) admits a unique local in time weak entropy solution on a time interval $I$. For all $t$ in $I$, we call $S_{t}$ the nonlinear

\footnotetext{
Keywords and phrases. Optimal control of the continuity equation, non-local flows.

${ }^{1}$ Department of Mathematics, Brescia University, Via Branze 38, 25133 Brescia, Italy. rinaldo@ing. unibs.it

2 RWTH Aachen University, Templergraben 55, 52056 Aachen, Germany.

${ }^{3}$ Université de Lyon, Université Lyon 1, École Centrale de Lyon, INSA de Lyon, CNRS UMR 5208, Institut Camille Jordan, 43 blvd. du 11 Novembre 1918, 69622 Villeurbanne Cedex, France.
} 
local semigroup that associates to the initial condition $\rho_{0}$ the solution $S_{t} \rho_{0}$ of (1.1) at time $t$. As in the standard case, $S_{t}$ turns out to be $\mathbf{L}^{1}$-Lipschitz.

Then, we look for the Gâteaux differentiability of the map $\rho_{0} \mapsto S_{t} \rho_{0}$, in any direction $r_{0}$ and for all $t \in I$. A weak Gâteaux differentiability of the semigroup generated by (1.1) is proved along any solution $\rho \in \mathcal{C}^{\mathbf{0}}\left(I ;\left(\mathbf{W}^{\mathbf{1 , 1}} \cap \mathbf{W}^{\mathbf{1}, \infty}\right)\left(\mathbb{R}^{N} ; \mathbb{R}\right)\right)$ and in any direction $r_{0} \in \mathbf{L}^{\mathbf{1}}\left(\mathbb{R}^{N} ; \mathbb{R}\right)$. Full differentiability follows under stronger assumptions. Moreover, the Gâteaux derivative of $S_{t}$ at $\rho_{0}$ in the direction $r_{0}$ is uniquely characterized as weak entropy solution to the following linear non-local Cauchy problem, that can be formally obtained by linearizing (1.1):

$$
\left\{\begin{array}{lrl}
\partial_{t} r+\operatorname{div}(r V(\rho)+\rho \mathrm{D} V(\rho)(r))=0 & (t, x) & \in I \times \mathbb{R}^{N} \\
r(0, x)=r_{0}(x) & x \in \mathbb{R}^{N}
\end{array}\right.
$$

where $\rho(t, x)=\left(S_{t} \rho_{0}\right)(x)$. Thus, also the well posedness of the nonlocal problem (1.2) needed to be proved, see Proposition 2.8. Remark that, in both (1.1) and (1.2), solutions are constructed in $\mathcal{C}^{\mathbf{0}}\left(I ; \mathbf{L}^{\mathbf{1}}\left(\mathbb{R}^{N} ; \mathbb{R}\right)\right)$. Therefore, we mostly refer to Kružkov solutions, see [21], Definition 1. Indeed, this definition of solutions is more demanding than that of weak solutions. Besides, it allows us to apply the results in [15], used in the subsequent part concerning the differentiability of solutions with respect to the initial datum. However, we note that in the case of the standard transport equation (5.2) and with the regularity conditions assumed below, the two notions of solution coincide, see Lemma 5.1.

We recall here the well known standard (i.e. local) situation: the semigroup generated by a conservation law is in general not differentiable in $\mathbf{L}^{1}$, not even in the scalar 1D case, see [9], Section 1. To cope with these issues, an entirely new differential structure was introduced in [9], and further developed in [6,10], also addressing optimal control problems, see [11,13]. However, the mere definition of the shift differential in the scalar 1D case takes alone about a page, see [13], p. 89-90. We refer to $[7,8,18,24,25]$ for further results and discussions about the scalar one-dimensional case.

Then, we introduce a cost function $\mathcal{J}: \mathcal{C}^{0}\left(I, \mathbf{L}^{1}\left(\mathbb{R}^{N} ; \mathbb{R}\right)\right) \rightarrow \mathbb{R}$ and, using the differentiability property given above, we find a necessary condition on the initial data $\rho_{0}$ in order to minimize $\mathcal{J}$ along the solutions to (1.1) associated to $\rho_{0}$. We stress that the present necessary conditions are obtained within the functional setting typical of scalar conservation laws, i.e. within $\mathbf{L}^{\mathbf{1}}$ and $\mathbf{L}^{\infty}$. No reflexivity property is ever used.

The paper is organized as follows. In Section 2, we state the main results of this paper. The differentiability is proved in Theorem 2.10 and applied to a control in supply chain management in Theorem 3.2. Sections 3 and 4 provide examples of models based on (1.1), and in Section 5 we give the detailed proofs of our results.

\section{Notation AND MAIN RESUlts}

Denote $\mathbb{R}_{+}=\left[0,+\infty\left[, \mathbb{R}_{+}^{*}=\right] 0,+\infty\left[\right.\right.$ and by $I$, respectively $I_{\mathrm{ex}}$, the interval $\left[0, T\left[\right.\right.$, respectively $\left[0, T_{\mathrm{ex}}[\right.$, for $T, T_{\text {ex }}>0$. The open ball in $\mathbb{R}^{N}$ centered at 0 with radius $\delta$ is denoted by $B(0, \delta)$. Furthermore, we introduce the norms:

$$
\begin{aligned}
\|v\|_{\mathbf{L}^{\infty}} & =\operatorname{ess}_{x \in \mathbb{R}^{N}}\|v(x)\|, & \|v\|_{\mathbf{W}^{1,1}} & =\|v\|_{\mathbf{L}^{1}}+\left\|\nabla_{x} v\right\|_{\mathbf{L}^{1}}, \\
\|v\|_{\mathbf{W}^{2, \infty}} & =\|v\|_{\mathbf{L}^{\infty}}+\left\|\nabla_{x} v\right\|_{\mathbf{L}^{\infty}}+\left\|\nabla_{x}^{2} v\right\|_{\mathbf{L}^{\infty}}, & \|v\|_{\mathbf{W}^{1, \infty}} & =\|v\|_{\mathbf{L}^{\infty}}+\left\|\nabla_{x} v\right\|_{\mathbf{L}^{\infty}}
\end{aligned}
$$

\subsection{Existence of a weak entropy solution to (1.1)}

Let $V: \mathbf{L}^{1}\left(\mathbb{R}^{N} ; \mathbb{R}\right) \rightarrow \mathcal{C}^{2}\left(\mathbb{R}^{N} ; \mathbb{R}^{N}\right)$ be a functional, not necessarily linear. A straightforward extension of [21], Definition 1, yields the following definition of weak solutions for (1.1). 
Definition 2.1. Fix $\rho_{0} \in \mathbf{L}^{\infty}\left(\mathbb{R}^{N} ; \mathbb{R}\right)$. A weak entropy solution to (1.1) on $I$ is a bounded measurable map $\rho \in \mathcal{C}^{0}\left(I ; \mathbf{L}_{\text {loc }}^{1}\left(\mathbb{R}^{N} ; \mathbb{R}\right)\right)$ which is a Kružkov solution to

$$
\left\{\begin{array}{l}
\partial_{t} \rho+\operatorname{div}(\rho w(t, x))=0 \quad \text { where } \quad w(t, x)=(V(\rho(t)))(x) . \\
\rho(0, x)=\rho_{0}(x)
\end{array} \quad\right. \text {. }
$$

Here, differently from [21], Definition 1, we require the full continuity in time.

Introduce the spaces

$$
\mathcal{X}=\left(\mathbf{L}^{\mathbf{1}} \cap \mathbf{L}^{\infty} \cap \mathbf{B V}\right)\left(\mathbb{R}^{N} ; \mathbb{R}\right) \quad \text { and } \quad \mathcal{X}_{\alpha}=\left(\mathbf{L}^{\mathbf{1}} \cap \mathbf{B V}\right)\left(\mathbb{R}^{N} ;[0, \alpha]\right) \text { for } \alpha>0
$$

both equipped with the $\mathbf{L}^{\mathbf{1}}$ distance. Obviously, $\mathcal{X}_{\alpha} \subset \mathbf{L}^{\infty}\left(\mathbb{R}^{N} ; \mathbb{R}\right)$ for all $\alpha>0$.

We pose the following assumptions on $V$, all of which are satisfied in the examples on supply chain and pedestrian flow as shown in Section 3 and Section 4, respectively.

(V1): There exists a function $C \in \mathcal{C}^{\mathbf{0}}\left(\mathbb{R}_{+} ; \mathbb{R}_{+}\right)$such that for all $\rho \in \mathbf{L}^{\mathbf{1}}\left(\mathbb{R}^{N}, \mathbb{R}\right)$,

$$
\begin{aligned}
& V(\rho) \in \mathbf{L}^{\infty}\left(\mathbb{R}^{N} ; \mathbb{R}^{N}\right), \\
&\left\|\nabla_{x} V(\rho)\right\|_{\mathbf{L}^{\infty}\left(\mathbb{R}^{N} ; \mathbb{R}^{N \times N}\right)} \leq C\left(\|\rho\|_{\mathbf{L}^{\infty}\left(\mathbb{R}^{N} ; \mathbb{R}\right)}\right), \\
&\left\|\nabla_{x} V(\rho)\right\|_{\mathbf{L}^{1}\left(\mathbb{R}^{N} ; \mathbb{R}^{N \times N}\right)} \leq C\left(\|\rho\|_{\mathbf{L}^{\infty}\left(\mathbb{R}^{N} ; \mathbb{R}\right)}\right), \\
&\left\|\nabla_{x}^{2} V(\rho)\right\|_{\mathbf{L}^{1}\left(\mathbb{R}^{N} ; \mathbb{R}^{N \times N \times N)}\right.} \leq C\left(\|\rho\|_{\mathbf{L}^{\infty}\left(\mathbb{R}^{N} ; \mathbb{R}\right)}\right) .
\end{aligned}
$$

There exists a function $C \in \mathcal{C}^{0}\left(\mathbb{R}_{+} ; \mathbb{R}_{+}\right)$such that for all $\rho_{1}, \rho_{2} \in \mathbf{L}^{\mathbf{1}}\left(\mathbb{R}^{N}, \mathbb{R}\right)$

$$
\begin{aligned}
&\left\|V\left(\rho_{1}\right)-V\left(\rho_{2}\right)\right\|_{\mathbf{L}^{\infty}\left(\mathbb{R}^{N} ; \mathbb{R}^{N}\right)} \leq C\left(\left\|\rho_{1}\right\|_{\mathbf{L}^{\infty}\left(\mathbb{R}^{N} ; \mathbb{R}\right)}\right)\left\|\rho_{1}-\rho_{2}\right\|_{\mathbf{L}^{1}\left(\mathbb{R}^{N} ; \mathbb{R}\right)}, \\
&\left\|\nabla_{x} V\left(\rho_{1}\right)-\nabla_{x} V\left(\rho_{2}\right)\right\|_{\mathbf{L}^{1}\left(\mathbb{R}^{N} ; \mathbb{R}^{N \times N}\right)} \leq C\left(\left\|\rho_{1}\right\|_{\mathbf{L}^{\infty}\left(\mathbb{R}^{N} ; \mathbb{R}\right)}\right)\left\|\rho_{1}-\rho_{2}\right\|_{\mathbf{L}^{1}\left(\mathbb{R}^{N} ; \mathbb{R}\right)} .
\end{aligned}
$$

(V2): There exists a function $C \in \mathcal{C}^{0}\left(\mathbb{R}_{+} ; \mathbb{R}_{+}\right)$such that for all $\rho \in \mathbf{L}^{\mathbf{1}}\left(\mathbb{R}^{N}, \mathbb{R}\right)$,

$$
\left\|\nabla_{x}^{2} V(\rho)\right\|_{\mathbf{L}^{\infty}\left(\mathbb{R}^{N} ; \mathbb{R}^{N \times N \times N)}\right.} \leq C\left(\|\rho\|_{\mathbf{L}^{\infty}\left(\mathbb{R}^{N} ; \mathbb{R}\right)}\right) .
$$

(V3): $V: \mathbf{L}^{\mathbf{1}}\left(\mathbb{R}^{N} ; \mathbb{R}\right) \rightarrow \mathcal{C}^{3}\left(\mathbb{R}^{N} ; \mathbb{R}^{N}\right)$ and there exists a function $C \in \mathcal{C}^{0}\left(\mathbb{R}_{+} ; \mathbb{R}_{+}\right)$such that for all $\rho \in$ $\mathbf{L}^{\mathbf{1}}\left(\mathbb{R}^{N}, \mathbb{R}\right)$,

$$
\left\|\nabla_{x}^{3} V(\rho)\right\|_{\mathbf{L}^{\infty}\left(\mathbb{R}^{N} ; \mathbb{R}^{N \times N \times N \times N)}\right.} \leq C\left(\|\rho\|_{\mathbf{L}^{\infty}\left(\mathbb{R}^{N} ; \mathbb{R}\right)}\right) .
$$

Condition (2.1) essentially requires that $V$ be a non local operator. Note that (V3) implies (V2). Existence of a solution to (1.1) (at least locally in time) can be proved under only assumption (V1), see Theorem 2.2. The stronger bounds on $V$ ensure additional regularity of the solution which is required later to derive the differentiability properties, see Proposition 2.5.

Theorem 2.2. Let (V1) hold. Then, for all $\alpha, \beta>0$ with $\beta>\alpha$, there exists a time $T(\alpha, \beta)>0$ such that for all $\rho_{0} \in \mathcal{X}_{\alpha}$, problem (1.1) admits a unique weak entropy solution $\rho \in \mathcal{C}^{0}\left([0, T(\alpha, \beta)] ; \mathcal{X}_{\beta}\right)$ in the sense of Definition 2.1. Moreover,

(1) $\|\rho(t)\|_{\mathbf{L}^{\infty}} \leq \beta$ for all $t \in[0, T(\alpha, \beta)]$.

(2) There exists a function $L \in \mathcal{C}^{\mathbf{0}}\left(\mathbb{R}_{+} ; \mathbb{R}_{+}\right)$such that for all $\rho_{0,1}, \rho_{0,2}$ in $\mathcal{X}_{\alpha}$, the corresponding solutions satisfy, for all $t \in[0, T(\alpha, \beta)]$,

$$
\left\|\rho_{1}(t)-\rho_{2}(t)\right\|_{\mathbf{L}^{1}} \leq L(t)\left\|\rho_{0,1}-\rho_{0,2}\right\|_{\mathbf{L}^{1}} .
$$


(3) There exists a constant $\mathcal{L}=\mathcal{L}(\beta)$ such that for all $\rho_{0} \in \mathcal{X}_{\alpha}$, the corresponding solution satisfies for all $t \in[0, T(\alpha, \beta)]$

$$
\operatorname{TV}(\rho(t)) \leq\left(\operatorname{TV}\left(\rho_{0}\right)+\mathcal{L} t\left\|\rho_{0}\right\|_{\mathbf{L}^{\infty}}\right) \mathrm{e}^{\mathcal{L} t} \quad \text { and } \quad\|\rho(t)\|_{\mathbf{L}^{\infty}} \leq\left\|\rho_{0}\right\|_{\mathbf{L}^{\infty}} \mathrm{e}^{\mathcal{L} t} .
$$

The above result is local in time. Indeed, as $\beta$ grows, $\mathcal{L}(\beta)$ may well grow, even unboundedly. Hence, both the total variation and the $\mathbf{L}^{\infty}$ norm of the solution may well blow up in finite time. To ensure global existence in time we need additional conditions on $V$ :

(A): $V$ is such that for all $\rho \in \mathbf{L}^{\mathbf{1}}\left(\mathbb{R}^{N} ; \mathbb{R}\right)$ and all $x \in \mathbb{R}^{N},(\operatorname{div} V(\rho))(x) \geq 0$.

(B): The function $C$ in (V1) is bounded, i.e. $C \in \mathbf{L}^{\infty}\left(\mathbb{R}_{+} ; \mathbb{R}_{+}\right)$.

Note that in the supply chain model discussed in Section 3, condition (A) applies. On the contrary, in the case of the pedestrian model in Section 4, iterating Theorem 2.2, we obtain the existence of solution up to time $\sum_{i} T\left(\alpha_{i}, \alpha_{i+1}\right)$. The latter turns out to be a convergent series, see Remark 5.5.

Lemma 2.3. Assume all assumptions of Theorem 2.2. Let also (A) hold. Then, for all $\alpha>0$, the set $\mathcal{X}_{\alpha}$ is invariant for (1.1), hence if the initial datum $\rho_{0}$ is such that $\left\|\rho_{0}\right\|_{\mathbf{L}^{\infty}\left(\mathbb{R}^{N} ; \mathbb{R}\right)} \leq \alpha$, then $\|\rho(t)\|_{\mathbf{L}^{\infty}\left(\mathbb{R}^{N} ; \mathbb{R}\right)} \leq \alpha$ as long as the solution $\rho(t)$ exists.

Condition (B), although it does not guarantee the boundedness of the solution, does ensure the global existence of the solution to (1.1).

Theorem 2.4. Let (V1) hold. Assume moreover that (A) or (B) hold. Then, there exists a unique semigroup $S: \mathbb{R}_{+} \times \mathcal{X} \rightarrow \mathcal{X}$ with the following properties:

(S1): For all $\rho_{0} \in \mathcal{X}$, the orbit $t \mapsto S_{t} \rho_{0}$ is a weak entropy solution to (1.1).

(S2): $S$ is $\mathbf{L}^{1}$-continuous in time, i.e. for all $\rho_{0} \in \mathcal{X}$, the map $t \mapsto S_{t} \rho_{0}$ is in $\mathcal{C}^{\mathbf{0}}\left(\mathbb{R}_{+} ; \mathcal{X}\right)$.

(S3): $S$ is $\mathbf{L}^{1}$-Lipschitz with respect to the initial datum, i.e. for a suitable positive $L \in \mathcal{C}^{0}\left(\mathbb{R}_{+} ; \mathbb{R}_{+}\right)$, for all $t \in \mathbb{R}_{+}$and all $\rho_{1}, \rho_{2} \in \mathcal{X}$,

$$
\left\|S_{t} \rho_{1}-S_{t} \rho_{2}\right\|_{\mathbf{L}^{1}\left(\mathbb{R}^{N} ; \mathbb{R}\right)} \leq L(t)\left\|\rho_{1}-\rho_{2}\right\|_{\mathbf{L}^{1}\left(\mathbb{R}^{N} ; \mathbb{R}\right)} .
$$

(S4): There exists a positive constant $\mathcal{L}$ such that for all $\rho_{0} \in \mathcal{X}$ and all $t \in \mathbb{R}_{+}$,

$$
\operatorname{TV}(\rho(t)) \leq\left(\operatorname{TV}\left(\rho_{0}\right)+\mathcal{L} t\left\|\rho_{0}\right\|_{\mathbf{L}^{\infty}\left(\mathbb{R}^{N} ; \mathbb{R}\right)}\right) \mathrm{e}^{\mathcal{L} t} .
$$

Higher regularity of the solutions of (1.1) can be proved under stronger bounds on $V$.

Proposition 2.5. Let (V1) and (V2) hold. With the same notations as in Theorem 2.2 , if $\rho_{0} \in \mathcal{X}_{\alpha}$, then

$$
\begin{aligned}
& \rho_{0} \in\left(\mathbf{W}^{\mathbf{1}, \mathbf{1}} \cap \mathbf{L}^{\infty}\right)\left(\mathbb{R}^{N} ; \mathbb{R}\right) \Longrightarrow \forall t \in[0, T(\alpha, \beta)], \quad \rho(t) \in \mathbf{W}^{\mathbf{1 , 1}}\left(\mathbb{R}^{N} ; \mathbb{R}\right), \\
& \rho_{0} \in \mathbf{W}^{\mathbf{1}, \infty}\left(\mathbb{R}^{N} ; \mathbb{R}\right) \quad \Longrightarrow \forall t \in[0, T(\alpha, \beta)], \quad \rho(t) \in \mathbf{W}^{\mathbf{1}, \infty}\left(\mathbb{R}^{N} ; \mathbb{R}\right),
\end{aligned}
$$

and there exists a positive constant $C=C(\beta)$ such that

$$
\|\rho(t)\|_{\mathbf{W}^{1,1}} \leq \mathrm{e}^{2 C t}\left\|\rho_{0}\right\|_{\mathbf{W}^{1,1}} \quad \text { and } \quad\|\rho(t)\|_{\mathbf{W}^{1, \infty}} \leq \mathrm{e}^{2 C t}\left\|\rho_{0}\right\|_{\mathbf{W}^{1, \infty}} .
$$

Furthermore, if $V$ also satisfies (V3), then

$$
\rho_{0} \in\left(\mathbf{W}^{\mathbf{2}, \mathbf{1}} \cap \mathbf{L}^{\infty}\right)\left(\mathbb{R}^{N} ;[\alpha, \beta]\right) \Longrightarrow \forall t \in[0, T(\alpha, \beta)], \quad \rho(t) \in \mathbf{W}^{\mathbf{2 , 1}}\left(\mathbb{R}^{N} ; \mathbb{R}\right)
$$

and for a suitable non-negative constant $C=C(\beta)$, we have the estimate

$$
\|\rho(t)\|_{\mathbf{W}^{2,1}} \leq \mathrm{e}^{C t}\left(2 \mathrm{e}^{C t}-1\right)^{2}\left\|\rho_{0}\right\|_{\mathbf{W}^{2,1}} .
$$

The proofs are deferred to Section 5 . 


\subsection{Differentiability}

This section is devoted to the differentiability of the semigroup $S$ (defined in Thm. 2.2) with respect to the initial datum $\rho_{0}$, according to the following notion. Recall first that a map $F: \mathbf{L}^{\mathbf{1}}\left(\mathbb{R}^{N} ; \mathbb{R}\right) \rightarrow \mathbf{L}^{\mathbf{1}}\left(\mathbb{R}^{N} ; \mathbb{R}\right)$ is strongly $\mathbf{L}^{\mathbf{1}}$ Gâteaux differentiable in any direction at $\rho_{0} \in \mathbf{L}^{\mathbf{1}}\left(\mathbb{R}^{N} ; \mathbb{R}\right)$ if there exists a continuous linear map $\mathrm{D} F\left(\rho_{0}\right): \mathbf{L}^{\mathbf{1}}\left(\mathbb{R}^{N} ; \mathbb{R}\right) \rightarrow \mathbf{L}^{\mathbf{1}}\left(\mathbb{R}^{N} ; \mathbb{R}\right)$ such that for all $r_{0} \in \mathbf{L}^{\mathbf{1}}\left(\mathbb{R}^{N} ; \mathbb{R}\right)$ and for any real sequence $\left(h_{n}\right)$ with $h_{n} \rightarrow 0$

$$
\frac{F\left(\rho_{0}+h_{n} r_{0}\right)-F\left(\rho_{0}\right)}{h_{n}} \stackrel{n \rightarrow \infty}{\rightarrow} \mathrm{D} F\left(\rho_{0}\right)\left(r_{0}\right) \quad \text { strongly in } \mathbf{L}^{\mathbf{1}}
$$

Besides proving the differentiability of the semigroup, we also characterize the differential. Formally, a sort of first order expansion of (1.1) with respect to the initial datum can be obtained through a standard linearizing procedure, which yields (1.2). Now, we rigorously show that the derivative of the semigroup in the direction $r_{0}$ is indeed the solution to (1.2) with initial condition $r_{0}$. To this aim, we need a forth and final condition on $V$.

(V4): There exists a function $K \in \mathcal{C}^{\mathbf{0}}\left(\mathbb{R}_{+} ; \mathbb{R}_{+}\right)$such that for all $\rho \in \mathbf{L}^{\mathbf{1}}\left(\mathbb{R}^{N} ; \mathbb{R}_{+}\right)$, for all $r \in \mathbf{L}^{\mathbf{1}}\left(\mathbb{R}^{N} ; \mathbb{R}\right)$,

$$
\begin{aligned}
\|V(\rho+r)-V(\rho)-\mathrm{D} V(\rho)(r)\|_{\mathbf{W}^{2, \infty}} & \leq K\left(\|\rho\|_{\mathbf{L}^{\infty}}+\|\rho+r\|_{\mathbf{L}^{\infty}}\right)\|r\|_{\mathbf{L}^{1}}^{2}, \\
\|\mathrm{D} V(\rho)(r)\|_{\mathbf{W}^{2, \infty}} & \leq K\left(\|\rho\|_{\mathbf{L}^{\infty}}\right)\|r\|_{\mathbf{L}^{1}} .
\end{aligned}
$$

Consider now system (1.2), where $\rho \in \mathcal{C}^{0}\left(I_{\mathrm{ex}}, \mathcal{X}\right)$ is a given function. We introduce a notion of solution for $(1.2)$ and give conditions which guarantee the existence of a solution.

Definition 2.6. Fix $r_{0} \in \mathbf{L}^{\infty}\left(\mathbb{R}^{N} ; \mathbb{R}\right)$. A function $r \in \mathcal{C}^{0}\left(I ; \mathbf{L}_{\text {loc }}^{1}\left(\mathbb{R}^{N} ; \mathbb{R}_{+}\right)\right)$, bounded and measurable, is a weak solution to $(1.2)$ if for any test function $\varphi \in \mathcal{C}_{c}^{\infty}\left(\stackrel{\circ}{I} \times \mathbb{R}^{N} ; \mathbb{R}\right)$

$$
\int_{0}^{T} \int_{\mathbb{R}^{N}}\left[r \partial_{t} \varphi+r a(t, x) \cdot \nabla_{x} \varphi-\operatorname{div} b(t, x) \varphi\right] \mathrm{d} x \mathrm{~d} t=0 \text { where } \begin{aligned}
& a=V(\rho) \\
& b=\rho \operatorname{DV}(\rho)(r)
\end{aligned}
$$

and $r(0)=r_{0}$ a.e. in $\mathbb{R}^{N}$.

We now extend the classical notion of Kružkov solution to the present non local setting.

Definition 2.7. Fix $r_{0} \in \mathbf{L}^{\infty}\left(\mathbb{R}^{N} ; \mathbb{R}_{+}\right)$. A function $r \in \mathcal{C}^{\mathbf{0}}\left(I ; \mathbf{L}_{\text {loc }}^{\mathbf{l}}\left(\mathbb{R}^{N} ; \mathbb{R}_{+}\right)\right)$, bounded and measurable, is a Kružkov solution to the nonlocal problem (1.2) if it is a Kružkov solution to

$$
\left\{\begin{array}{l}
\partial_{t} r+\operatorname{div}(r a(t, x)+b(t, x))=0 \\
r(0, x)=r_{0}(x)
\end{array} \quad \text { where } a=V(\rho) \text { and } b=\rho \mathrm{D} V(\rho)(r)\right.
$$

In other words, $r$ is a Kružkov solution to $(1.2)$ if for all $k \in \mathbb{R}$ and for any test function $\varphi \in \mathcal{C}_{c}^{\infty}\left(\stackrel{\circ}{I} \times \mathbb{R}^{N} ; \mathbb{R}_{+}\right)$

$$
\begin{aligned}
& \int_{0}^{T} \int_{\mathbb{R}^{N}}\left[(r-k) \partial_{t} \varphi+(r-k) V(\rho) \cdot \nabla_{x} \varphi-\operatorname{div}(k V(\rho)+\rho \mathrm{D} V(\rho)(r)) \varphi\right] \operatorname{sgn}(r-k) \mathrm{d} x \mathrm{~d} t \geq 0 \\
& \text { and } \lim _{t \rightarrow 0^{+}} \int_{B(0, \delta)}\left|r(t)-r_{0}\right| \mathrm{d} x=0 \quad \text { for all } \delta>0 .
\end{aligned}
$$

Condition (V4) ensures that if $\rho \in \mathbf{W}^{\mathbf{1}, \mathbf{1}}\left(\mathbb{R}^{N} ; \mathbb{R}\right)$, then $\mathrm{D} V(\rho)(r) \in \mathcal{C}^{\mathbf{2}}\left(\mathbb{R}^{N} ; \mathbb{R}^{N}\right)$ and hence for all $t \geq 0$, the map $x \mapsto \rho(t, x) \mathrm{D} V(\rho(t))(r(t, x))$ is in $\mathbf{W}^{\mathbf{1}, \mathbf{1}}\left(\mathbb{R}^{N} ; \mathbb{R}\right)$, so that the integral above is meaningful.

Proposition 2.8. Let (V1), (V4) hold. Fix $\rho \in \mathcal{C}^{\mathbf{0}}\left(I_{\mathrm{ex}} ; \mathbf{L}^{\mathbf{1}}\left(\mathbb{R}^{N} ; \mathbb{R}\right)\right)$ such that $\rho(t) \in\left(\mathbf{W}^{\mathbf{1}, \infty} \cap \mathbf{W}^{\mathbf{1}, \mathbf{1}}\right)\left(\mathbb{R}^{N} ; \mathbb{R}\right)$ for all $t \in I_{\mathrm{ex}}$. Then, for all $r_{0} \in\left(\mathbf{L}^{\mathbf{1}} \cap \mathbf{L}^{\infty}\right)\left(\mathbb{R}^{N} ; \mathbb{R}\right)$ there exists a unique weak entropy solution to $(1.2)$ 
in $\mathcal{C}^{\mathbf{0}}\left(I_{\mathrm{ex}} ; \mathbf{L}^{\mathbf{1}}\left(\mathbb{R}^{N} ; \mathbb{R}\right)\right)$ and for all time $t \in I_{\mathrm{ex}}$, with $C=C\left(\|\rho\|_{\mathbf{L}^{\infty}\left([0, t] \times \mathbb{R}^{N} ; \mathbb{R}\right)}\right)$ as in (V1) and $K=$ $K\left(\|\rho\|_{\mathbf{L}^{\infty}\left([0, t] \times \mathbb{R}^{N} ; \mathbb{R}\right)}\right)$ as in $(\mathbf{V} \mathbf{4})$

$$
\begin{aligned}
\|r(t)\|_{\mathbf{L}^{1}} & \leq \mathrm{e}^{K t\|\rho\|_{\mathbf{L}^{\infty}\left(I ; \mathbf{W}^{1,1}\right)}} \mathrm{e}^{C t}\left\|r_{0}\right\|_{\mathbf{L}^{1}} \\
\|r(t)\|_{\mathbf{L}^{\infty}} & \leq \mathrm{e}^{C t}\left\|r_{0}\right\|_{\mathbf{L}^{\infty}}+K t \mathrm{e}^{2 C t} \mathrm{e}^{K t\|\rho\|_{\mathbf{L}^{\infty}\left(I ; \mathbf{W}^{1,1}\right)}\|\rho\|_{\mathbf{L}^{\infty}\left(I ; \mathbf{W}^{1, \infty}\right)}\left\|r_{0}\right\|_{\mathbf{L}^{1}} .}
\end{aligned}
$$

If (V2) holds, $\rho \in \mathbf{L}^{\infty}\left(I_{\mathrm{ex}} ;\left(\mathbf{W}^{\mathbf{1}, \infty} \cap \mathbf{W}^{\mathbf{2 , 1}}\right)\left(\mathbb{R}^{N} ; \mathbb{R}\right)\right)$ and $r_{0} \in\left(\mathbf{W}^{\mathbf{1 , 1}} \cap \mathbf{L}^{\infty}\right)\left(\mathbb{R}^{N} ; \mathbb{R}\right)$, then for all $t \in I_{\mathrm{ex}}$, $r(t) \in \mathbf{W}^{\mathbf{1}, \mathbf{1}}\left(\mathbb{R}^{N} ; \mathbb{R}\right)$ and

$$
\|r(t)\|_{\mathbf{W}^{1,1}} \leq\left(1+C^{\prime} t\right) \mathrm{e}^{2 C^{\prime} t}\left\|r_{0}\right\|_{\mathbf{W}^{1,1}}+K t(1+C t) \mathrm{e}^{4 C^{\prime} t}\left\|r_{0}\right\|_{\mathbf{L}^{1}}\|\rho\|_{\mathbf{L}^{\infty}\left(I ; \mathbf{W}^{2,1}\right)}
$$

where $C^{\prime}=\max \left\{C, K\|\rho\|_{\mathbf{L}^{\infty}\left(I_{\mathrm{ex}} ; \mathbf{W}^{1,1}\left(\mathbb{R}^{N} ; \mathbb{R}\right)\right)}\right\}$.

Under (V1) and (V4), the following mild differentiability result can be proved.

Proposition 2.9. Let (V1) and (V4) hold. Let $\rho_{0} \in\left(\mathbf{W}^{\mathbf{1}, \infty} \cap \mathbf{W}^{\mathbf{1 , 1}}\right)\left(\mathbb{R}^{N} ; \mathbb{R}\right)$ and $r_{0} \in \mathcal{X}_{1}$. Then, there exist $h_{*}>0$ and $T_{*}=T_{*}\left(\left\|\rho_{0}\right\|_{\mathbf{L}^{\infty}}\right)$, such that for all $h \in\left[0, h_{*}\right]$ the solution $\rho$ to (1.1) and the solution $\rho_{h}$ to

$$
\left\{\begin{array}{lc}
\partial_{t} \rho_{h}+\operatorname{div}\left(\rho_{h} V\left(\rho_{h}\right)\right)=0 & (t, x) \in \mathbb{R}_{+} \times \mathbb{R}^{N} \\
\rho_{h}(0, x)=\rho_{0}(x)+h r_{0}(x) & x \in \mathbb{R}^{N}
\end{array}\right.
$$

are defined for all $t \in\left[0, T_{*}\right]$. Moreover, if there exists an $r \in \mathbf{L}^{\mathbf{1}}\left(\left[0, T_{*}\right] \times \mathbb{R}^{N} ; \mathbb{R}\right)$ such that $\left(\rho_{h}-\rho\right) / h^{h \rightarrow 0}{ }^{0}$, then $r$ is a distributional solution to (1.2), i.e. it satisfies (2.2).

Below, we consider the following stronger hypothesis, under which we derive a result of strong Gâteaux differentiability and uniqueness of the derivative.

(V5): There exists a function $K \in \mathcal{C}^{\mathbf{0}}\left(\mathbb{R}_{+} ; \mathbb{R}_{+}\right)$such that for all $\rho, \tilde{\rho} \in \mathbf{L}^{\mathbf{1}}\left(\mathbb{R}^{N} ; \mathbb{R}\right)$

$$
\|\operatorname{div}(V(\tilde{\rho})-V(\rho)-\mathrm{D} V(\rho)(\tilde{\rho}-\rho))\|_{\mathbf{L}^{1}} \leq K\left(\|\rho\|_{\mathbf{L}^{\infty}}+\|\tilde{\rho}\|_{\mathbf{L}^{\infty}}\right)\left(\|\tilde{\rho}-\rho\|_{\mathbf{L}^{1}}\right)^{2}
$$

and the map $r \rightarrow \operatorname{div} \mathrm{D} V(\rho)(r)$ is a bounded linear operator on $\mathbf{L}^{\mathbf{1}}\left(\mathbb{R}^{N} ; \mathbb{R}\right) \rightarrow \mathbf{L}^{\mathbf{1}}\left(\mathbb{R}^{N} ; \mathbb{R}\right)$, i.e. for all $\rho, r \in \mathbf{L}^{\mathbf{1}}\left(\mathbb{R}^{N} ; \mathbb{R}\right)$

$$
\|\operatorname{div}(\operatorname{DV}(\rho)(r))\|_{\mathbf{L}^{1}\left(\mathbb{R}^{N} ; \mathbb{R}\right)} \leq K\left(\|\rho\|_{\mathbf{L}^{\infty}\left(\mathbb{R}^{N} ; \mathbb{R}\right)}\right)\|r\|_{\mathbf{L}^{1}\left(\mathbb{R}^{N} ; \mathbb{R}\right)} .
$$

Theorem 2.10. Let (V1), (V3), (V4) and (V5) hold. Let $\rho_{0} \in\left(\mathbf{W}^{\mathbf{1}, \infty} \cap \mathbf{W}^{\mathbf{2}, \mathbf{1}}\right)\left(\mathbb{R}^{N} ; \mathbb{R}\right), r_{0} \in\left(\mathbf{W}^{\mathbf{1 , 1}} \cap\right.$ $\left.\mathbf{L}^{\infty}\right)\left(\mathbb{R}^{N} ; \mathbb{R}\right)$, and denote $T_{\mathrm{ex}}$ the time of existence of the solution of (1.1) with initial condition $\rho_{0}$. Then, for all time $t \in I_{\mathrm{ex}}$ the local semigroup defined in Theorem 2.2 is strongly $\mathbf{L}^{1}$ Gâteaux differentiable in the direction $r_{0}$. The derivative $\mathrm{D} S_{t}\left(\rho_{0}\right)\left(r_{0}\right)$ of $S_{t}$ at $\rho_{0}$ in the direction $r_{0}$ is

$$
\mathrm{D} S_{t}\left(\rho_{0}\right)\left(r_{0}\right)=\Sigma_{t}^{\rho_{0}}\left(r_{0}\right)
$$

where $\Sigma^{\rho_{0}}$ is the linear application generated by the Kružkov solution to (1.2), where $\rho=S_{t} \rho_{0}$, then for all $t \in I_{\mathrm{ex}}$.

\subsection{Necessary optimality conditions for problems governed by (1.1)}

Aiming at necessary optimality conditions for non linear functionals defined on the solutions to (1.1), we prove the following chain rule formula. 
Proposition 2.11. Let $T>0$ and $I=\left[0, T\left[\right.\right.$. Assume that $f \in \mathcal{C}^{\mathbf{1}, \mathbf{1}}\left(\mathbb{R} ; \mathbb{R}_{+}\right), \psi \in \mathbf{L}^{\infty}\left(I \times \mathbb{R}^{N} ; \mathbb{R}\right)$ and that $S: I \times\left(\mathbf{L}^{1} \cap \mathbf{L}^{\infty}\right)\left(\mathbb{R}^{N} ; \mathbb{R}\right) \rightarrow\left(\mathbf{L}^{1} \cap \mathbf{L}^{\infty}\right)\left(\mathbb{R}^{N} ; \mathbb{R}\right)$ is strongly $\mathbf{L}^{1}$ Gâteaux differentiable. For all $t \in I$, let

$$
J\left(\rho_{0}\right)=\int_{\mathbb{R}^{N}} f\left(S_{t} \rho_{0}\right) \psi(t, x) \mathrm{d} x .
$$

Then, $J$ is strongly $\mathbf{L}^{\infty}$ Gâteaux differentiable in any direction $r_{0} \in\left(\mathbf{W}^{\mathbf{1}, \mathbf{1}} \cap \mathbf{L}^{\infty}\right)\left(\mathbb{R}^{N} ; \mathbb{R}\right)$. Moreover,

$$
\mathrm{D} J\left(\rho_{0}\right)\left(r_{0}\right)=\int_{\mathbb{R}^{N}} f^{\prime}\left(S_{t} \rho_{0}\right) \Sigma_{t}^{\rho_{0}}\left(r_{0}\right) \psi(t, x) \mathrm{d} x .
$$

Proof. Since $\left|f\left(\rho_{h}\right)-f(\rho)-f^{\prime}(\rho)\left(\rho_{h}-\rho\right)\right| \leq \operatorname{Lip}\left(f^{\prime}\right)\left|\rho_{h}-\rho\right|^{2}$, we have

$$
\begin{aligned}
& \mid \frac{J\left(\rho_{0}+h r_{0}\right)-J\left(\rho_{0}\right)}{h}-\int_{\mathbb{R}^{N}} f^{\prime}\left(S_{t} \rho_{0}\right) \mathrm{D} S_{t}\left(\rho_{0}\right)\left(r_{0}\right) \psi(t, x) \mathrm{d} x \mid \\
& \leq \int_{\mathbb{R}^{N}}\left|f^{\prime}\left(S_{t} \rho_{0}\right)\right| \frac{S_{t}\left(\rho_{0}+h r_{0}\right)-S_{t}\left(\rho_{0}\right)}{h}-\operatorname{D} S_{t}\left(\rho_{0}\right)\left(r_{0}\right)|| \psi(t, x) \mid \mathrm{d} x \\
& \quad+\operatorname{Lip}\left(f^{\prime}\right) \frac{1}{|h|} \int_{\mathbb{R}^{N}}\left|S_{t}\left(\rho_{0}+h r_{0}\right)-S_{t}\left(\rho_{0}\right)\right|^{2}|\psi(t, x)| \mathrm{d} x .
\end{aligned}
$$

The strong Gâteaux differentiability of $S_{t}$ in $\mathbf{L}^{1}$ then yields

$$
\int_{\mathbb{R}^{N}}\left|f^{\prime}\left(S_{t} \rho_{0}\right)\right| \frac{S_{t}\left(\rho_{0}+h r_{0}\right)-S_{t}\left(\rho_{0}\right)}{h}-\mathrm{D} S_{t}\left(\rho_{0}\right)\left(r_{0}\right)|| \psi(t, x) \mid \mathrm{d} x=o(1) \quad \text { as } h \rightarrow 0
$$

thanks to $S_{t} \rho_{0} \in \mathbf{L}^{\infty}$ and to the local boundedness of $f^{\prime}$. Furthermore,

$$
\begin{gathered}
S_{t}\left(\rho_{0}\right), S_{t}\left(\rho_{0}+h r_{0}\right) \in \mathbf{L}^{\infty} \\
\frac{1}{h}\left(S_{t}\left(\rho_{0}+h r_{0}\right)-S_{t}\left(\rho_{0}\right)\right) \stackrel{h \rightarrow 0}{\longrightarrow} \mathrm{D} S_{t}\left(\rho_{0}\right)\left(r_{0}\right) \text { pointwise a.e. } \\
S_{t}\left(\rho_{0}+h r_{0}\right)-S_{t}\left(\rho_{0}\right) \stackrel{h \rightarrow 0}{\longrightarrow} 0 \text { pointwise a.e. }
\end{gathered}
$$

the Dominated Convergence Theorem ensures that the higher order term in the latter expansion tend to 0 as $h \rightarrow 0$.

The above result can be easily extended. First, to more general (non linear) functionals $J\left(\rho_{0}\right)=\mathcal{J}\left(S_{t} \rho_{0}\right)$, with $\mathcal{J}: \mathcal{X} \rightarrow \mathbb{R}_{+}$such that for all $\rho \in \mathcal{X}$ there exists a continuous linear application $\mathrm{D} \mathcal{J}(\rho): \mathcal{X} \rightarrow \mathbb{R}$ such that for all $\rho, r \in \mathcal{X}$ :

$$
\left|\frac{\mathcal{J}(\rho+h r)-\mathcal{J}(\rho)}{h}-\mathrm{D} \mathcal{J}(\rho)(r)\right| \stackrel{h \rightarrow 0}{\longrightarrow} 0 .
$$

Secondly, to functionals of the type

$$
J\left(\rho_{0}\right)=\int_{0}^{T} \int_{\mathbb{R}^{N}} f\left(S_{t} \rho_{0}\right) \psi(t, x) \mathrm{d} x \mathrm{~d} t \quad \text { or } \quad J\left(\rho_{0}\right)=\int_{0}^{T} \mathcal{J}\left(S_{t} \rho_{0}\right) \mathrm{d} t .
$$

This generalization, however, is immediate and we omit the details.

Once the differentiability result above is available, a necessary condition of optimality is straightforward. 
Proposition 2.12. Let $f \in \mathcal{C}^{1,1}\left(\mathbb{R} ; \mathbb{R}_{+}\right)$and $\psi \in \mathbf{L}^{\infty}\left(I_{\mathrm{ex}} \times \mathbb{R}^{N} ; \mathbb{R}\right)$. Assume that $S: I \times\left(\mathbf{L}^{\mathbf{1}} \cap \mathbf{L}^{\infty}\right)\left(\mathbb{R}^{N} ; \mathbb{R}\right) \rightarrow$ $\left(\mathbf{L}^{\mathbf{1}} \cap \mathbf{L}^{\infty}\right)\left(\mathbb{R}^{N} ; \mathbb{R}\right)$ is strongly $\mathbf{L}^{\mathbf{1}}$ Gâteaux differentiable. Define $J$ as in $(2.4)$. If $\rho_{0} \in\left(\mathbf{L}^{\mathbf{1}} \cap \mathbf{L}^{\infty}\right)\left(\mathbb{R}^{N} ; \mathbb{R}\right)$ solves the problem

$$
\text { find } \min _{\rho_{0}} \mathcal{J}(\rho) \text { subject to }\{\rho \text { is solution to }(1.1)\}
$$

then, for all $r_{0} \in\left(\mathbf{L}^{1} \cap \mathbf{L}^{\infty}\right)\left(\mathbb{R}^{N} ; \mathbb{R}\right)$

$$
\int_{\mathbb{R}^{N}} f^{\prime}\left(S_{t} \rho_{0}\right) \Sigma_{t}^{\rho_{0}} r_{0} \psi(t, x) \mathrm{d} x=0 .
$$

\section{DEMAND TRACKING PROBlEMS FOR SUPPly CHAins}

Recently, Armbruster et al. [4], introduced a continuum model to simulate the average behavior of highly re-entrant production systems at an aggregate level appearing, for instance, in large volume semiconductor production line. The factory is described by the density of products $\rho(t, x)$ at stage $x$ of the production at a time $t$. Typically, see $[1,4,20]$, the production velocity $V$ is a given smooth function of the total load $\int_{0}^{1} \rho(t, x) \mathrm{d} x$, for example

$$
v(u)=v_{\max } /(1+u) \quad \text { and } \quad V(\rho)=v\left(\int_{0}^{1} \rho(t, s) \mathrm{d} s\right) .
$$

The full model, given by (1.1)-(3.1) with $N=1$, fits in the present framework.

Proposition 3.1. Let $v \in \mathcal{C}^{1}([0,1] ; \mathbb{R})$. Then, the functional $V$ defined as in (3.1) satisfies (A), (V1), (V2), (V3). Moreover, if $v \in \mathcal{C}^{2}([0,1] ; \mathbb{R})$, then $V$ satisfies also $(\mathbf{V} 4)$ and (V5).

The proof is deferred to Section 5.4.

The supply chain model with $V$ given by (3.1) satisfies (V1) to (V5) and (A). Therefore, Theorem 2.4 applies and, in particular, the set $[0,1]$ is invariant yielding global well posedness. By Theorem 2.10, the semigroup $S_{t} \rho_{0}$ is Gâteaux differentiable in any direction $r_{0}$ and the differential is given by the solution to (1.2).

Note that the velocity is constant across the entire system at any time. In fact, in a real world factory, all parts move through the factory with the same speed. While in a serial production line, speed through the factory is dependent on all items and machines downstream, in a highly re-entrant factory this is not the case. Since items must visit machines more than once, including machines at the beginning of the production process, their speed through factory is determined by the total number of parts both upstream and downstream from them. Such re-entrant production is characteristic for semiconductor production lines. Typically, the output of the whole factory over a longer timescale, e.g. following a seasonal demand pattern or ramping up or down a new product, can be controlled by prescribing the inflow density to a factory $\rho(t, x=0)=\lambda(t)$. The influx should be chosen in order to achieve either of the following objective goals [4]:

(1) Minimize the mismatch between the outflow and a demand rate target $\mathrm{d}(t)$ over a fixed time period (demand tracking problem). This is modelled by the cost functional $\frac{1}{2} \int_{0}^{T}(\mathrm{~d}(t)-\rho(1, t))^{2} \mathrm{~d} t$.

(2) Minimize the mismatch between the total number of parts that have left the factory and the desired total number of parts over a fixed time period $\mathrm{d}(t)$. The backlog of a production system at a given time $t$ is defined as the total number of items that have been demanded minus the total number of items that have left the factory up to that time. Backlog can be negative or positive, with a negative backlog corresponding to overproduction and a positive backlog corresponding to a shortage. This problem is modeled by $\frac{1}{2} \int_{0}^{T}\left(\int_{0}^{t} \mathrm{~d}(\tau) u-\rho(1, \tau) \mathrm{d} \tau\right)^{2} \mathrm{~d} t$.

In both cases we are interested in the influx $\lambda(t)$. A numerical integration of this problem has been studied in [22]. In order to apply the previous calculus we reformulate the optimization problem for the influx density 
$\lambda(t)=\rho_{0}(-t)$ where $\rho_{0}$ is the solution to a minimization problem for

$$
\begin{aligned}
& J_{1}\left(\rho_{0}\right)=\frac{1}{2} \int_{0}^{1}\left(d(x)-S_{T} \rho_{0}(x)\right)^{2} \mathrm{~d} x \\
& J_{2}\left(\rho_{0}\right)=\frac{1}{2} \int_{0}^{1}\left(\int_{0}^{x}\left(d(\xi)-S_{T} \rho_{0}(\xi)\right) \mathrm{d} \xi\right)^{2} \mathrm{~d} x,
\end{aligned}
$$

respectively, where $S_{t} \rho_{0}$ is the solution to (1.1) and (3.1). Clearly, $J_{1}$ and $J_{2}$ satisfy the assumptions imposed in the previous section. The assertions of Proposition 2.12 then state necessary optimality conditions, which we summarize in the theorem below.

Theorem 3.2. Let $T>0$ be given. Let the assumptions of Proposition 3.1 hold. Let $\rho_{0} \in\left(\mathbf{W}^{\mathbf{1}, \infty} \cap \mathbf{W}^{\mathbf{2}, \mathbf{1}}\right)(\mathbb{R} ; \mathbb{R})$ be a minimizer of $J_{1}$ as defined in (3.2), with $S$ being the semigroup generated by (1.1)-(3.1). Then, for all $r_{0} \in\left(\mathbf{W}^{\mathbf{1}, \infty} \cap \mathbf{W}^{\mathbf{2}, \mathbf{1}}\right)(\mathbb{R} ; \mathbb{R})$ we have

$$
\begin{gathered}
\int_{0}^{1}(d(x)-\rho(T, x)) r(T, x) \mathrm{d} x=0, \quad \text { where } \\
\partial_{t} r+\partial_{x}\left(v_{\max } \frac{\left(r \int_{0}^{1} \rho \mathrm{d} x+\rho \int_{0}^{1} r \mathrm{~d} x\right)}{\left(1+\int_{0}^{1} \rho \mathrm{d} x\right)^{2}}\right)=0, \quad r(0, x)=r_{0}(x) .
\end{gathered}
$$

The latter Cauchy problem is in the form (1.2) and Proposition 2.8 proves its well posedness. The latter proof is deferred to Section 5.4.

\section{A MODEL FOR PEDESTRIAN FLOW}

Macroscopic models for pedestrian movements are based on the continuity equation, see $[12,14,16,19]$, possibly together with a second equation, as in [17]. In these models, pedestrians are assumed to instantaneously adjust their (vector) speed according to the crowd density at their position. The analytical construction in Section 2 allows to consider the more realistic situation of pedestrian deciding their speed according to the local mean density at their position. We are thus led to consider (1.1) with

$$
V(\rho)=v(\rho * \eta) \vec{v}
$$

where

$$
\eta \in \mathcal{C}_{c}^{2}\left(\mathbb{R}^{2} ;[0,1]\right) \text { has support } \operatorname{spt} \eta \subseteq B(0,1) \text { and }\|\eta\|_{\mathbf{L}^{1}}=1,
$$

so that $(\rho * \eta)(x)$ is an average of the values attained by $\rho$ in $B(x, 1)$. Here, $\vec{v}=\vec{v}(x)$ is the given direction of the motion of the pedestrian at $x \in \mathbb{R}^{2}$. Then, the presence of boundaries, obstacles or other geometric constraint can be described through $\vec{v}$, see [16].

Note that condition (A) does not allow any increase in the crowd density. Hence, it may not hold when describing, say, the evacuation through a narrow exit. Therefore, in general, for this example we have only a local in time solution by Theorem 2.2 .

As in the preceding example, first we state the hypotheses that guarantee assumptions (V1) to (V5).

Proposition 4.1. Let $V$ be defined in (4.1) and $\eta$ be as in (4.2).

(1) If $v \in \mathcal{C}^{2}(\mathbb{R} ; \mathbb{R})$ and $\vec{v} \in\left(\mathcal{C}^{\mathbf{2}} \cap \mathbf{W}^{\mathbf{2}, \mathbf{1}}\right)\left(\mathbb{R}^{2} ; \mathbb{S}^{1}\right)$, then $V$ satisfies (V1) and (V2).

(2) If moreover $v \in \mathcal{C}^{3}(\mathbb{R} ; \mathbb{R}), \vec{v} \in \mathcal{C}^{3}\left(\mathbb{R}^{2} ; \mathbb{R}^{2}\right)$ and $\eta \in \mathcal{C}^{3}\left(\mathbb{R}^{2} ; \mathbb{R}\right)$ then $V$ satisfies (V3).

(3) If moreover $v \in \mathcal{C}^{4}(\mathbb{R} ; \mathbb{R}), \vec{v} \in \mathcal{C}^{2}\left(\mathbb{R}^{2} ; \mathbb{R}^{2}\right)$ and $\eta \in \mathcal{C}^{2}\left(\mathbb{R}^{2} ; \mathbb{R}\right)$, then $V$ satisfies (V4) and (V5). 
The proof is deferred to Section 5.4.

A typical problem in the management of pedestrian flows consists in keeping the crowd density $\rho(t, x)$ below a given threshold, say $\hat{\rho}$, in particular in a sensible compact region $\Omega$. To this aim, it is natural to introduce a cost functional of the type

where

$$
J\left(\rho_{0}\right)=\int_{0}^{T} \int_{\mathbb{R}^{N}} f\left(S_{t} \rho_{0}(x)\right) \psi(t, x) \mathrm{d} x \mathrm{~d} t
$$

$(\mathbf{f}): f \in \mathcal{C}^{\mathbf{1}, \mathbf{1}}\left(\mathbb{R} ; \mathbb{R}_{+}\right), f(\rho)=0$ for $\rho \in[0, \hat{\rho}], f(\rho)>0$ and $f^{\prime}(\rho)>0$ for $\rho>\hat{\rho}$.

$(\psi): \psi \in \mathcal{C}^{\infty}\left([0, T] \times \mathbb{R}^{N} ;[0,1]\right)$, with $\operatorname{spt} \psi(t)=\Omega$, is a smooth approximation of the characteristic function of the compact set $\Omega$, with $\Omega \neq \emptyset$.

Section 2.3 then applies, yielding the following necessary condition for optimality.

Theorem 4.2. Let $T>0$ and the assumptions of (1)-(3) in Proposition 4.1 hold, together with (f) and $(\psi)$. Let $\rho_{0} \in\left(\mathbf{W}^{\mathbf{1}, \infty} \cap \mathbf{W}^{\mathbf{2}, \mathbf{1}}\right)(\mathbb{R} ; \mathbb{R})$ be a minimizer of $J$ as defined in (4.3), with $S$ being the semigroup generated by (1.1)-(4.1). Then, for all $r_{0} \in\left(\mathbf{W}^{\mathbf{1}, \infty} \cap \mathbf{W}^{\mathbf{2}, \mathbf{1}}\right)(\mathbb{R} ; \mathbb{R})$, $\rho_{0}$ satisfies $(2.5)$.

The proof is deferred to Section 5.4.

Consider the problem of evacuating a meeting room. Then, the optimal $\rho_{0}$ corresponds to the best distribution of people during meetings, so that the room is evacuated in the minimal time in case of need.

\section{Detailed PRoOfs}

Below, we denote by $W_{N}$ the Wallis integral

$$
W_{N}=\int_{0}^{\pi / 2}(\cos \alpha)^{N} \mathrm{~d} \alpha
$$

\subsection{A lemma on the transport equation}

In what follows, a key role will be played by the transport equation

$$
\left\{\begin{array}{l}
\partial_{t} r+\operatorname{div}(r w(t, x))=R(t, x) \\
r(0, x)=r_{0}(x)
\end{array}\right.
$$

The next lemma is similar to other results in recent literature, see for instance [2]. It provides the existence and uniqueness of solutions to (5.2) with the present regularity conditions, showing also that the concepts of weak and Kružkov solution here coincide, see also [26].

Lemma 5.1. Let $T>0$, so that $I=[0, T[$, and $w$ be such that

$$
\begin{array}{lrl}
w \in \mathcal{C}^{0}\left(I \times \mathbb{R}^{N} ; \mathbb{R}^{N}\right) & w(t) & \in \mathcal{C}^{1}\left(\mathbb{R}^{N} ; \mathbb{R}^{N}\right) \quad \forall t \in I \\
w \in \mathbf{L}^{\infty}\left(I \times \mathbb{R}^{N} ; \mathbb{R}^{N}\right) & \nabla_{x} w \in \mathbf{L}^{\infty}\left(I \times \mathbb{R}^{N} ; \mathbb{R}^{N \times N}\right) .
\end{array}
$$

Assume that $R \in \mathbf{L}^{\infty}\left(I ; \mathbf{L}^{\mathbf{1}}\left(\mathbb{R}^{N} ; \mathbb{R}\right)\right) \cap \mathbf{L}^{\infty}\left(I \times \mathbb{R}^{N} ; \mathbb{R}\right)$ and $r_{0} \in\left(\mathbf{L}^{\mathbf{1}} \cap \mathbf{L}^{\infty}\right)\left(\mathbb{R}^{N} ; \mathbb{R}\right)$. Then,

(1) the function $r$ defined by

$$
\begin{aligned}
r(t, x)= & r_{0}(X(0 ; t, x)) \exp \left(-\int_{0}^{t} \operatorname{div} w(\tau, X(\tau ; t, x)) \mathrm{d} \tau\right) \\
& +\int_{0}^{t} R(\tau, X(\tau ; t, x)) \exp \left(-\int_{\tau}^{t} \operatorname{div} w(u, X(u ; t, x)) \mathrm{d} u\right) \mathrm{d} \tau,
\end{aligned}
$$


where $t \mapsto X\left(t ; t_{0}, x_{0}\right)$ is the solution to the Cauchy problem

$$
\left\{\begin{array}{l}
\frac{\mathrm{d} \chi}{\mathrm{d} t}=w(t, \chi) \\
\chi\left(t_{0}\right)=x_{0}
\end{array}\right.
$$

is such that $r \in \mathcal{C}^{0}\left(I ; \mathbf{L}^{\mathbf{1}}\left(\mathbb{R}^{N} ; \mathbb{R}\right)\right)$;

(2) the function $r$, as defined by (5.4), is a Kružkov solution to (5.2);

(3) any weak solution to (5.2) coincides with $r$ as defined in (5.4).

Recall that, in the present case, [21], Definition 1, amounts to define Kružkov solution to (5.2) a function $r \in \mathbf{L}^{\infty}\left(I ; \mathbf{L}_{\text {loc }}^{1}\left(\mathbb{R}^{N} ; \mathbb{R}\right)\right)$, continuous from the right in time, such that for all $k \in \mathbb{R}$, for all test function $\varphi \in \mathcal{C}_{c}^{\infty}(] 0, T\left[\times \mathbb{R}^{N} ; \mathbb{R}_{+}\right)$

$$
\int_{0}^{T} \int_{\mathbb{R}^{N}}\left[(r-k)\left(\partial_{t} \varphi+w \cdot \nabla_{x} \varphi\right)+(R-k \operatorname{div} w) \varphi\right] \operatorname{sgn}(r-k) \mathrm{d} x \mathrm{~d} t \geq 0
$$

and such that for almost every $t \in[0, T]$, for any $\delta>0$,

$$
\lim _{t \rightarrow 0} \int_{B(0, \delta)}\left|r(t, x)-r_{0}(x)\right| \mathrm{d} x=0 .
$$

On the other hand, by weak solution to (5.3) we mean a map $r \in \mathcal{C}^{0}\left(I ; \mathbf{L}^{\mathbf{1}}\left(\mathbb{R}^{N} ; \mathbb{R}\right)\right) \cap \mathbf{L}^{\infty}\left(I \times \mathbb{R}^{N} ; \mathbb{R}\right)$ that satisfies (5.3) in distributional sense. Remark that as an immediate corollary of Lemma 5.1, we obtain that any weak solution to (5.2) is also a Kružkov solution and is represented by (5.4).

Note that the expression (5.4) is formally justified integrating (5.2) along the characteristics (5.5) and obtaining

$$
\frac{\mathrm{d}}{\mathrm{d} t}(r(t, \chi(t)))+r(t, \chi(t)) \operatorname{div} w(t, \chi(t))=R(t, \chi(t)) .
$$

Recall for later use that the flow $X=X\left(t ; t_{0}, x_{0}\right)$ generated by $(5.5)$ can be used to introduce the change of variable $y=X(0 ; t, x)$, so that $x=X(t ; 0, y)$, due to standard properties of the Cauchy problem (5.5). Denote by $J(t, y)=\operatorname{det}\left(\nabla_{y} X(t ; 0, y)\right)$ the Jacobian of this change of variables. Then, $J$ satisfies the equation

$$
\frac{\mathrm{d} J(t, y)}{\mathrm{d} t}=\operatorname{div} w(t, X(t ; 0, y)) J(t, y)
$$

with initial condition $J(0, y)=1$. Hence $J(t, y)=\exp \left(\int_{0}^{t} \operatorname{div} w(\tau, X(\tau ; 0, y)) \mathrm{d} \tau\right)$ which, in particular, implies $J(t, y)>0$ for all $t \in I, y \in \mathbb{R}^{N}$.

Proof of Lemma 5.1.

(1) Let $R_{n} \in \mathbf{L}^{\infty}\left(I ; \mathcal{C}^{1}\left(\mathbb{R}^{N} ; \mathbb{R}\right)\right)$ and $r_{0, n} \in \mathcal{C}^{\mathbf{1}}\left(\mathbb{R}^{N} ; \mathbb{R}\right)$ approximate $R$ and $r_{0}$ in the sense

$$
\left\|R_{n}-R\right\|_{\mathbf{L}^{\infty}\left(I ; \mathbf{L}^{1}\left(\mathbb{R}^{N} ; \mathbb{R}\right)\right)} \rightarrow 0 \quad \text { and } \quad\left\|r_{0, n}-r_{n}\right\|_{\mathbf{L}^{1}\left(\mathbb{R}^{N} ; \mathbb{R}\right)} \rightarrow 0 \quad \text { as } \quad n \rightarrow+\infty .
$$

Call $r_{n}$ the corresponding quantity as given by (5.4). Then, by (5.4), also

$$
\left\|r_{n}-r\right\|_{\mathbf{L}^{\infty}\left(I ; \mathbf{L}^{1}\left(\mathbb{R}^{N} ; \mathbb{R}\right)\right)} \rightarrow 0 \quad \text { as } \quad n \rightarrow+\infty
$$

so that $r \in \mathbf{L}^{\infty}\left(I ; \mathbf{L}^{\mathbf{1}}\left(\mathbb{R}^{N} ; \mathbb{R}\right)\right)$. Concerning continuity in time, simply note that, by (5.4), $r_{n} \in$ $\mathcal{C}^{\mathbf{0}}\left(I ; \mathbf{L}^{\mathbf{1}}\left(\mathbb{R}^{N} ; \mathbb{R}\right)\right)$ and $r$ is the uniform limit of the $r_{n}$. 
(2) The boundedness requirement follows from (5.4):

$$
\|r(t)\|_{\mathbf{L}^{\infty}\left(\mathbb{R}^{N} ; \mathbb{R}\right)} \leq\left(\left\|r_{0}\right\|_{\mathbf{L}^{\infty}\left(\mathbb{R}^{N} ; \mathbb{R}\right)}+t\|R\|_{\mathbf{L}^{\infty}\left(I \times \mathbb{R}^{N} ; \mathbb{R}\right)}\right) \mathrm{e}^{t\|\operatorname{div} w\|_{\mathbf{L}^{\infty}\left(I \times \mathbb{R}^{N} ; \mathbb{R}\right)}}
$$

Let $k \in \mathbb{R}$ and $\varphi \in \mathcal{C}_{\boldsymbol{c}}^{\infty}\left(\stackrel{I}{I} \times \mathbb{R}^{N} ; \mathbb{R}_{+}\right)$. Then, according to [21], Definition 1, we prove (5.6) for $r$ given as in (5.4). By (5.8), the semigroup property of $X$ and denoting $\mathcal{R}(t, y)=\int_{0}^{t} R(\tau, X(\tau ; 0, y)) J(\tau, y) \mathrm{d} \tau$, so that $\mathcal{R}$ is $\mathbf{W}^{\mathbf{1 , 1}}$ in time, we get

$$
\begin{aligned}
\int_{0}^{+\infty} \int_{\mathbb{R}^{N}}\left[(r-k)\left(\partial_{t} \varphi+w \cdot \nabla_{x} \varphi\right)+(R-k \operatorname{div} w) \varphi\right] \operatorname{sgn}(r-k) \mathrm{d} x \mathrm{~d} t=\int_{0}^{+\infty} \int_{\mathbb{R}^{N}}\left[\left(\frac{r_{0}(y)}{J(t, y)}+\frac{\mathcal{R}(t, y)}{J(t, y)}-k\right)\right. \\
\quad \times\left(\partial_{t} \varphi(t, X(t ; 0, y))+w(t, X(t ; 0, y)) \cdot \nabla_{x} \varphi(t, X(t ; 0, y))\right) \\
\quad+(R(t, X(t ; 0, y))-k \operatorname{div}(w(t, X(t ; 0, y)))) \varphi(t, X(t ; 0, y))] \\
\quad \times \operatorname{sgn}\left(\frac{r_{0}(y)}{J(t, y)}+\frac{\mathcal{R}(t, y)}{J(t, y)}-k\right) J(t, y) \mathrm{d} y \mathrm{~d} t \\
=\int_{0}^{+\infty} \int_{\mathbb{R}^{N}}\left[r_{0}(y) \frac{\mathrm{d}}{\mathrm{d} t} \varphi(t, X(t ; 0, y))-k J(t, y) \frac{\mathrm{d}}{\mathrm{d} t} \varphi(t, X(t ; 0, y))\right. \\
\left.\quad-k \varphi(t, X(t ; 0, y)) \frac{\mathrm{d}}{\mathrm{d} t} J(t, y)+\frac{\mathrm{d}}{\mathrm{d} t}(\mathcal{R}(t, y) \varphi(t, X(t ; 0, y)))\right] \\
=\int_{0}^{+\infty} \int_{\mathbb{R}^{N}} \frac{\mathrm{d}}{\mathrm{d} t}\left(\left(r_{0}(y)+\mathcal{R}(t, y)-k J(t, y)\right) \varphi(t, X(t ; 0, y))\right) \\
\quad \times \operatorname{sgn}\left(r_{0}(y)+\mathcal{R}(t, y)-k J(t, y)\right) \mathrm{d} y \mathrm{~d} t \\
=\int_{0}^{+\infty} \int_{\mathbb{R}^{N}} \frac{\mathrm{d}}{\mathrm{d} t}\left(\left|r_{0}(y)+\mathcal{R}(t, y)-k J(t, y)\right| \varphi(t, X(t ; 0, y))\right) \mathrm{d} y \mathrm{~d} t \\
=0 .
\end{aligned}
$$

Finally, (5.7) holds by the continuity proved above.

(3) Let $r$ be a weak solution to (5.3). It is enough to consider the case $R=0$ and $r_{0}=0$, thanks to the linearity of (5.3). Then, fix $\tau \in] 0, T]$, choose any $\varphi \in \mathcal{C}^{1}\left(I ; \mathcal{C}_{c}^{1}\left(\mathbb{R}^{N} ; \mathbb{R}\right)\right)$ and let $\beta_{\varepsilon} \in \mathcal{C}^{1}(I ; \mathbb{R})$ such that $\beta_{\varepsilon}(t)=1$ for $t \in[\varepsilon, \tau-\varepsilon], \beta_{\varepsilon}^{\prime}(t) \in[0,2 / \varepsilon]$ for $t \in[0, \varepsilon], \beta_{\varepsilon}^{\prime}(t) \in[-2 / \varepsilon, 0]$ for $t \in[\tau-\varepsilon, \tau]$ and $\beta(t)=0$ for $t \in[\tau, T]$. By the definition of weak solution,

$$
\begin{aligned}
0= & \int_{0}^{\tau} \int_{\mathbb{R}^{N}}\left(r \partial_{t} \varphi+r w \cdot \nabla \varphi\right) \beta_{\varepsilon} \mathrm{d} x \mathrm{~d} t+\int_{0}^{\varepsilon} \beta_{\varepsilon}^{\prime} \int_{\mathbb{R}^{N}} r \varphi \mathrm{d} x \mathrm{~d} t+\int_{\tau-\varepsilon}^{\tau} \int_{\mathbb{R}^{N}} r \varphi \beta_{\varepsilon}^{\prime} \mathrm{d} x \mathrm{~d} t \\
= & \int_{0}^{\tau} \beta_{\varepsilon} \int_{\mathbb{R}^{N}}\left(r \partial_{t} \varphi+r w \cdot \nabla \varphi\right) \mathrm{d} x \mathrm{~d} t+\int_{0}^{1} \varepsilon \beta_{\varepsilon}^{\prime}(\varepsilon t) \int_{\mathbb{R}^{N}} r(\varepsilon t, x) \varphi(\varepsilon t, x) \mathrm{d} x \mathrm{~d} t \\
& \quad+\int_{\tau-\varepsilon}^{\tau} \beta_{\varepsilon}^{\prime}(t) \int_{\mathbb{R}^{N}} r(t, x) \varphi(t, x) \mathrm{d} x \mathrm{~d} t .
\end{aligned}
$$

Consider the three terms separately. As $\varepsilon \rightarrow 0$, the first one converges to $\int_{\mathbb{R}^{N}} \int_{0}^{\tau} r\left(\partial_{t} \varphi+w \cdot \nabla \varphi\right) \mathrm{d} t \mathrm{~d} x$. Consider the second summand, by the Dominated Convergence Theorem, it tends to 0 , since $r_{0}=0$. 
Passing to the latter term, note that:

$$
\left|\int_{\tau-\varepsilon}^{\tau} \beta_{\varepsilon}^{\prime}(t) \int_{\mathbb{R}^{N}} r(t, x) \varphi(t, x) \mathrm{d} x \mathrm{~d} t+\int_{\mathbb{R}^{N}} r(\tau, x) \varphi(\tau, x) \mathrm{d} x\right|=\left|\int_{\tau-\varepsilon}^{\tau} \beta_{\varepsilon}^{\prime}(t) \int_{\mathbb{R}^{N}}(r(t, x) \varphi(t, x)-r(\tau, x) \varphi(\tau, x)) \mathrm{d} x \mathrm{~d} t\right|
$$

by the continuity of $r$ in time and the smoothness of $\varphi$.

Therefore, choose any $\eta \in \mathcal{C}_{c}^{1}\left(\mathbb{R}^{N} ; \mathbb{R}\right)$ and define $\varphi$ as the backward solution to $\partial_{t} \varphi+w \cdot \nabla \varphi=0$ with $\varphi(\tau)=\eta$. Then,

$$
\begin{aligned}
0 & =\int_{\mathbb{R}^{N}} \int_{0}^{\tau} r\left(\partial_{t} \varphi+w \cdot \nabla \varphi\right) \mathrm{d} t \mathrm{~d} x-\int_{\mathbb{R}^{N}} r(\tau, x) \varphi(\tau, x) \mathrm{d} x \\
& =\int_{\mathbb{R}^{N}} r(\tau, x) \varepsilon(x) \mathrm{d} x
\end{aligned}
$$

proving that $r(\tau)$, and hence $r$, vanishes identically.

\subsection{Proof of Theorem $\mathbf{2 . 2}$}

Corollary 5.2. In the same setting of Lemma 5.1, if $R=0$ then:

$$
\begin{aligned}
& \rho_{0} \geq 0 \text { for a.e. } x \Rightarrow \rho(t) \geq 0 \text { for a.e. } x \text { and for all } t \in I, \\
& \operatorname{div} w \geq 0 \text { for a.e. }(t, x) \Rightarrow\|\rho(t)\|_{\mathbf{L}^{\infty}} \leq\left\|\rho_{0}\right\|_{\mathbf{L}^{\infty}} \text { for all } t \in I, \\
&\|\rho(t)\|_{\mathbf{L}^{\infty}\left(\mathbb{R}^{N} ; \mathbb{R}\right)} \leq\left\|\rho_{0}\right\|_{\mathbf{L}^{\infty}\left(\mathbb{R}^{N} ; \mathbb{R}\right)} \exp \left(t\|\operatorname{div} w\|_{\mathbf{L}^{\infty}\left([0, t] \times \mathbb{R}^{N} ; \mathbb{R}\right)}\right) .
\end{aligned}
$$

The proof follows directly from Lemma 5.1 using in particular (5.4).

Lemma 5.3. In the same setting of Lemma 5.1, if $R=0$ and also

$$
\operatorname{div} w \in \mathbf{L}^{\infty}\left(I ; \mathbf{L}^{\mathbf{1}}\left(\mathbb{R}^{N} ; \mathbb{R}\right)\right) \quad \text { and } \quad \nabla_{x} \operatorname{div} w \in \mathbf{L}^{\infty}\left(I ; \mathbf{L}^{\mathbf{1}}\left(\mathbb{R}^{N} ; \mathbb{R}^{N}\right)\right)
$$

then, setting

$$
\kappa_{0}=N W_{N}(2 N+1)\left\|\nabla_{x} w\right\|_{\mathbf{L}^{\infty}\left(I \times \mathbb{R}^{N} ; \mathbb{R}^{N \times N}\right)} \quad \text { and } \quad \kappa=2 N\left\|\nabla_{x} w\right\|_{\mathbf{L}^{\infty}\left(I \times \mathbb{R}^{N} ; \mathbb{R}^{N \times N}\right)}
$$

we have the following bound on the total variation:

$$
\begin{aligned}
\operatorname{TV}(\rho(t)) \leq & \operatorname{TV}\left(\rho_{0}\right) \mathrm{e}^{\kappa_{0} t} \\
& +N W_{N} \int_{0}^{t} \mathrm{e}^{\kappa_{0}(t-s)} \int_{\mathbb{R}^{N}} \mathrm{e}^{s\|\operatorname{div} w\|_{\mathbf{L}^{\infty}}}\left\|\nabla_{x} \operatorname{div} w(s, x)\right\| \mathrm{d} x \mathrm{~d} s\left\|\rho_{0}\right\|_{\mathbf{L}^{\infty}}
\end{aligned}
$$

where $\left\|\nabla_{x} \operatorname{div} w(s, x)\right\|$ is the usual Euclidean norm of a vector in $\mathbb{R}^{N}$. 
Let $\rho_{1}, \rho_{2}$ be the solutions of (5.2) associated to $w_{1}, w_{2}$ with $R_{1}=R_{2}=0$ and with initial conditions $\rho_{1,0}$, $\rho_{2,0}$ in $\mathcal{X}$. Then

$$
\begin{aligned}
\|\left(\rho_{1}-\right. & \left.\rho_{2}\right)(t)\left\|_{\mathbf{L}^{1}} \leq \mathrm{e}^{\kappa t}\right\| \rho_{1,0}-\rho_{2,0}\left\|_{\mathbf{L}^{1}}+\frac{\mathrm{e}^{\kappa_{0} t}-\mathrm{e}^{\kappa t}}{\kappa_{0}-\kappa} \mathrm{TV}\left(\rho_{1,0}\right)\right\| w_{1}-w_{2} \|_{\mathbf{L}^{\infty}} \\
& +N W_{N} \int_{0}^{t} \frac{\mathrm{e}^{\kappa_{0}(t-s)}-\mathrm{e}^{\kappa(t-s)}}{\kappa_{0}-\kappa} \int_{\mathbb{R}^{N}} \mathrm{e}^{s\|\operatorname{div} w\|_{\mathbf{L}^{\infty}}}\left\|\nabla_{x} \operatorname{div} w_{1}(s, x)\right\| \mathrm{d} x \mathrm{~d} s \\
& \times\left\|\rho_{1,0}\right\|_{\mathbf{L}^{\infty}}\left\|w_{1}-w_{2}\right\|_{\mathbf{L}^{\infty}} \\
& +\int_{0}^{t} \mathrm{e}^{\kappa(t-s)} \mathrm{e}^{s\|\operatorname{div} w\|_{\mathbf{L}^{\infty}}} \int_{\mathbb{R}^{N}}\left|\operatorname{div}\left(w_{1}-w_{2}\right)(s, x)\right| \mathrm{d} x \mathrm{~d} s\left\|\rho_{1,0}\right\|_{\mathbf{L}^{\infty}},
\end{aligned}
$$

where

$$
\kappa_{0}=N W_{N}(2 N+1)\left\|\nabla_{x} w_{1}\right\|_{\mathbf{L}^{\infty}\left(I \times \mathbb{R}^{N} ; \mathbb{R}^{N \times N}\right)} \quad \text { and } \quad \kappa=2 N\left\|\nabla_{x} w_{1}\right\|_{\mathbf{L}^{\infty}\left(I \times \mathbb{R}^{N} ; \mathbb{R}^{N \times N}\right)} .
$$

Proof. The bound (5.12) follows from [15], Theorem 2.5, the hypotheses on $w$ being satisfied thanks to (5.3) and (5.11). More precisely, we do not have here the $\mathcal{C}^{2}$ regularity in time as required in [15], Theorem 2.5, but going through the proof of this result, we can see that only the continuity in time of the flow function $f(t, x, r)=r w(t, x)$ is necessary. Indeed, time derivatives of $f$ appear in the proof of [15], Theorem 2.5, when we bound the terms $J_{t}$ and $L_{t}$, see [15], between (4.18) and (4.19). However, the use of the Dominated Convergence Theorem allows to prove that $J_{t}$ and $L_{t}$ converge to zero when $\eta$ goes to 0 without any use of time derivatives. The continuity in times follows from [15], Remark 2.4, thanks to (5.11) of $w$ and the bound on the total variation.

Similarly, the stability estimate (5.13) is based on [15], Theorem 2.6. Indeed, we use once again a flow that is only $\mathcal{C}^{0}$ instead of $\mathcal{C}^{2}$ in time. Besides, in the proof of [15], Theorem 2.6, the $\mathbf{L}^{\infty}$ bound into the integral term in [15], Theorem 2.6, can be taken only in space, keeping time fixed. With this provision, the proof of (5.13) is exactly the same as that in [15], so we do not reproduce it here. The same estimate is thus obtained, except that the $\mathbf{L}^{\infty}$ bound of the integral term is taken only in space.

Lemma 5.4. In the same setting of Lemma 5.1 if $R=0$ and (5.11) holds, together with

$$
\left\|\nabla_{x}^{2} w\right\|_{\mathbf{L}^{\infty}\left(I \times \mathbb{R}^{N} ; \mathbb{R}^{N \times N \times N}\right)} \leq C
$$

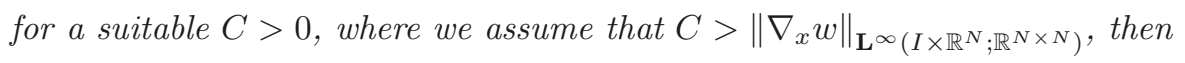

$$
\begin{aligned}
& \rho_{0} \in \mathbf{W}^{\mathbf{1 , 1}}\left(\mathbb{R}^{N} ; \mathbb{R}\right) \Rightarrow\left\{\begin{array}{l}
\rho(t) \in \mathbf{W}^{\mathbf{1}, \mathbf{1}}\left(\mathbb{R}^{N} ; \mathbb{R}\right) \quad \text { for all } t \in I \\
\|\rho(t)\|_{\mathbf{W}^{1,1}} \leq \mathrm{e}^{2 C t}\left\|\rho_{0}\right\|_{\mathbf{W}^{1,1}},
\end{array}\right. \\
& \rho_{0} \in \mathbf{W}^{\mathbf{1}, \infty}\left(\mathbb{R}^{N} ; \mathbb{R}\right) \Rightarrow\left\{\begin{array}{l}
\rho(t) \in \mathbf{W}^{\mathbf{1}, \infty}\left(\mathbb{R}^{N} ; \mathbb{R}\right) \quad \text { for all } t \in I \\
\|\rho(t)\|_{\mathbf{W}^{1, \infty}} \leq \mathrm{e}^{2 C t}\left\|\rho_{0}\right\|_{\mathbf{W}^{1, \infty}} .
\end{array}\right.
\end{aligned}
$$

If moreover $\left\|\nabla_{x}^{3} w\right\|_{\mathbf{L}^{\infty}\left(I \times \mathbb{R}^{N} ; \mathbb{R}^{N \times N \times N \times N)}\right.} \leq C$ then $\rho_{0} \in \mathbf{W}^{\mathbf{2}, \mathbf{1}}\left(\mathbb{R}^{N} ; \mathbb{R}\right)$ implies that $\rho(t) \in \mathbf{W}^{\mathbf{2}, \mathbf{1}}\left(\mathbb{R}^{N} ; \mathbb{R}\right)$ for all $t \in I$ and

$$
\|\rho(t)\|_{\mathbf{W}^{2,1}} \leq(1+C t)^{2} \mathrm{e}^{3 C t}\left\|\rho_{0}\right\|_{\mathbf{W}^{2,1}} .
$$


Proof. The $\mathbf{W}^{\mathbf{1 , 1}}$ and $\mathbf{W}^{\mathbf{1}, \infty}$ bounds follow from the representation (5.4) with $R=0$, noting that $\left\|\nabla_{x} X\right\|_{\mathbf{L}^{\infty}} \leq \mathrm{e}^{C t}$. Indeed,

$$
\begin{aligned}
\nabla_{x} X(t ; 0, x) & =\mathbf{I d}+\int_{0}^{t} \nabla_{x} w(\tau ; X(\tau ; 0, x)) \nabla_{x} X(\tau ; 0, x) \mathrm{d} \tau, \text { hence } \\
\left\|\nabla_{x} X(t ; 0, x)\right\| & \leq 1+\int_{0}^{t}\left\|\nabla_{x} w(\tau ; X(\tau ; 0, x))\right\|\left\|\nabla_{x} X(\tau ; 0, x)\right\| \mathrm{d} \tau \\
& \leq 1+\int_{0}^{t} C\left\|\nabla_{x} X(\tau ; 0, x)\right\| \mathrm{d} \tau
\end{aligned}
$$

and a direct application of Gronwall Lemma gives the desired bound. Hence, we obtain

$$
\|\nabla \rho(t)\|_{\mathbf{L}^{\infty}} \leq\left(\mathrm{e}^{2 C t}-\mathrm{e}^{C t}\right)\left\|\rho_{0}\right\|_{\mathbf{L}^{\infty}}+\mathrm{e}^{2 C t}\left\|\nabla \rho_{0}\right\|_{\mathbf{L}^{\infty}}
$$

and consequently

$$
\|\rho(t)\|_{\mathbf{W}^{1, \infty}} \leq \mathrm{e}^{2 C t}\left\|\rho_{0}\right\|_{\mathbf{W}^{1, \infty}} .
$$

Similarly, the $\mathbf{W}^{\mathbf{1}, \mathbf{1}}$ estimate also comes from (5.4).

To obtain the $\mathbf{W}^{\mathbf{2}, \mathbf{1}}$ bound (5.14), apply (5.4) and Gronwall Lemma to get $\left\|\nabla_{x}^{2} X\right\|_{\mathbf{L}^{\infty}} \leq \mathrm{e}^{2 C t}-\mathrm{e}^{C t}$. Using the estimates above, together with

$$
\left\|\nabla^{2} \rho(t)\right\|_{\mathbf{L}^{1}} \leq\left(2 \mathrm{e}^{2 C t}-3 \mathrm{e}^{C t}+1\right) \mathrm{e}^{C t}\left\|\rho_{0}\right\|_{\mathbf{L}^{1}}+3\left(\mathrm{e}^{C t}-1\right) \mathrm{e}^{2 C t}\left\|\nabla \rho_{0}\right\|_{\mathbf{L}^{1}}+\mathrm{e}^{3 C t}\left\|\nabla^{2} \rho_{0}\right\|_{\mathbf{L}^{1}},
$$

we obtain $\|\rho(t)\|_{\mathrm{W}^{2,1}} \leq\left(2 \mathrm{e}^{C t}-1\right)^{2} \mathrm{e}^{C t}\left\|\rho_{0}\right\|_{\mathrm{W}^{2,1}}$, concluding the proof.

We use now these tools in order to obtain the existence of a solution for (1.1).

Proof of Theorem 2.2. Fix $\alpha, \beta>0$ with $\beta>\alpha$. Let $T(\alpha, \beta)=(\ln (\beta / \alpha)) / C(\beta)$, with $C$ as in (V1). Define the map

$$
\mathcal{Q}: \begin{aligned}
\mathcal{C}^{0}\left(I ; \mathcal{X}_{\beta}\right) & \rightarrow \mathcal{C}^{0}\left(I ; \mathcal{X}_{\beta}\right) \\
\sigma & \mapsto \quad \rho
\end{aligned}
$$

where $I=[0, T(\alpha, \beta)[$ and $\rho$ is the Kružkov solution to

$$
\left\{\begin{array}{l}
\partial_{t} \rho+\operatorname{div}(\rho w)=0 \\
\rho(0, x)=\rho_{0}(x)
\end{array} \quad \text { with } \quad \begin{array}{c}
w=V(\sigma) \\
\rho_{0} \in \mathcal{X}_{\alpha}
\end{array}\right.
$$

Assumptions (V1) imply the hypotheses on $w$ necessary in Corollary 5.2 and Lemma 5.3. Therefore, a solution $\rho$ to (5.15) exists, is unique and in $\mathcal{X}$. Note that by (5.10), by the choice of $T(\alpha, \beta)$ and by (V1), we have $\|\rho(t)\|_{\mathbf{L}^{\infty}} \leq \beta$ and hence $\mathcal{Q}$ is well defined.

Fix $\sigma_{1}, \sigma_{2}$ in $\mathcal{C}^{0}\left(I ; \mathcal{X}_{\beta}\right)$. Call $w_{i}=V\left(\sigma_{i}\right)$ and $\rho_{i}$ the corresponding solutions. With the same notations of [15], Theorem 2.6, we let

$$
\kappa_{0}=N W_{N}(2 N+1)\left\|\nabla_{x} w_{1}\right\|_{\mathbf{L}^{\infty}\left(I \times \mathbb{R}^{N} ; \mathbb{R}^{N \times N}\right)}, \quad \kappa=2 N\left\|\nabla_{x} w_{1}\right\|_{\mathbf{L}^{\infty}\left(I \times \mathbb{R}^{N} ; \mathbb{R}^{N \times N}\right)} .
$$

Note that by $(5.1)$

$$
\frac{\kappa_{0}}{\kappa} \geq\left(N+\frac{1}{2}\right) \int_{0}^{\pi / 2}\left(1-\frac{2}{\pi} x\right)^{N} \mathrm{~d} x=\frac{\pi}{2}\left(1-\frac{1}{2(N+1)}\right) \geq \frac{3 \pi}{8}>1
$$

hence $\kappa_{0}>\kappa$. Then, by $(\mathbf{V} 1)$, we obtain a bound on $\kappa_{0}$. Indeed,

$$
\left\|\nabla_{x} V\left(\sigma_{1}\right)\right\|_{\mathbf{L}^{\infty}\left(I \times \mathbb{R}^{N} ; \mathbb{R}^{N \times N}\right)} \leq C\left(\left\|\sigma_{1}\right\|_{\mathbf{L}^{\infty}\left(I \times \mathbb{R}^{N} ; \mathbb{R}\right)}\right),
$$


and since $\sigma_{1} \in \mathcal{C}^{0}\left(I ; \mathcal{X}_{\beta}\right)$, finally $\kappa_{0} \leq N W_{N}(2 N+1) C(\beta)$. Let us denote

$$
C=C(\beta) \quad \text { and } \quad C^{\prime}=N W_{N}(2 N+1) C(\beta) .
$$

Again, (V1) implies the following uniform bounds on all $\sigma_{1}, \sigma_{2} \in \mathcal{C}^{0}\left(I ; \mathcal{X}_{\beta}\right)$ :

$$
\begin{aligned}
&\left\|\nabla_{x}^{2} V\left(\sigma_{1}\right)\right\|_{\mathbf{L}^{\infty}\left(I ; \mathbf{L}^{1}\left(\mathbb{R}^{N} ; \mathbb{R}^{N \times N \times N}\right)\right)} \leq C, \\
&\left\|V\left(\sigma_{1}\right)-V\left(\sigma_{2}\right)\right\|_{\mathbf{L}^{\infty}\left(I \times \mathbb{R}^{N} ; \mathbb{R}^{N}\right)} \leq C\left\|\sigma_{1}-\sigma_{2}\right\|_{\mathbf{L}^{\infty}\left(I ; \mathbf{L}^{1}\left(\mathbb{R}^{N} ; \mathbb{R}\right)\right)}, \\
&\left\|\operatorname{div}\left(V\left(\sigma_{1}\right)-V\left(\sigma_{2}\right)\right)\right\|_{\mathbf{L}^{\infty}\left(I ; \mathbf{L}^{1}\left(\mathbb{R}^{N} ; \mathbb{R}\right)\right)} \leq C\left\|\sigma_{1}-\sigma_{2}\right\|_{\mathbf{L}^{\infty}\left(I ; \mathbf{L}^{1}\left(\mathbb{R}^{N} ; \mathbb{R}\right)\right)} .
\end{aligned}
$$

Thus, we can apply [15], Theorem 2.6. We get, for all $t \in I$,

$$
\begin{aligned}
\left\|\left(\rho_{1}-\rho_{2}\right)(t)\right\|_{\mathbf{L}^{1}} \leq & C t \mathrm{e}^{C^{\prime} t} \operatorname{TV}\left(\rho_{0}\right)\left\|\sigma_{1}-\sigma_{2}\right\|_{\mathbf{L}^{\infty}\left([0, t] ; \mathbf{L}^{1}\right)} \\
& +C^{2} N W_{N} \mathrm{e}^{C t}\left\|\rho_{0}\right\|_{\mathbf{L}^{\infty}}\left\|\sigma_{1}-\sigma_{2}\right\|_{\mathbf{L}^{\infty}\left([0, t] ; \mathbf{L}^{1}\right)} \int_{0}^{t}(t-s) \mathrm{e}^{C^{\prime}(t-s)} \mathrm{d} s \\
& +C \mathrm{e}^{C t}\left\|\rho_{0}\right\|_{\mathbf{L}^{\infty}} \int_{0}^{t} \mathrm{e}^{C^{\prime}(t-s)}\left\|\left(\sigma_{1}-\sigma_{2}\right)(s)\right\|_{\mathbf{L}^{1}} \mathrm{~d} s .
\end{aligned}
$$

Therefore, we obtain the following Lipschitz estimate:

$$
\left\|\mathcal{Q}\left(\sigma_{1}\right)-\mathcal{Q}\left(\sigma_{2}\right)\right\|_{\mathbf{L}^{\infty}\left(I ; \mathbf{L}^{1}\right)} \leq C T \mathrm{e}^{C^{\prime} T}\left[\mathrm{TV}\left(\rho_{0}\right)+\left(N W_{N} C T+1\right) \mathrm{e}^{C T}\left\|\rho_{0}\right\|_{\mathbf{L}^{\infty}}\right]\left\|\sigma_{1}-\sigma_{2}\right\|_{\mathbf{L}^{\infty}\left(I ; \mathbf{L}^{1}\right)} .
$$

Here we introduce the strictly increasing function

$$
f(T)=C T \mathrm{e}^{C^{\prime} T}\left[\mathrm{TV}\left(\rho_{0}\right)+\left(N W_{N} C T+1\right) \mathrm{e}^{C T}\left\|\rho_{0}\right\|_{\mathbf{L}^{\infty}}\right]
$$

and we remark that $f(T) \rightarrow 0$ when $T \rightarrow 0$. Choose now $T_{1}>0$ so that $f\left(T_{1}\right)=1 / 2$. Banach Contraction Principle now ensures the existence and uniqueness of a solution $\rho^{*}$ to $(1.1)$ on $[0, \bar{T}]$ in the sense of Definition 2.1, with $\bar{T}=\min \left\{T(\alpha, \beta), T_{1}\right\}$. In fact, if $T_{1}<T(\alpha, \beta)$, we can prolongate the solution until time $T(\alpha, \beta)$. Indeed, if we take $\rho^{*}\left(T_{1}\right)$ as initial condition, we remark that $\left\|\rho^{*}\left(T_{1}\right)\right\|_{\mathbf{L}^{\infty}} \leq\left\|\rho_{0}\right\|_{\mathbf{L}^{\infty}} \mathrm{e}^{C(\beta) T_{1}}$. Consequently, the solution of $(5.15)$ on $\left[T_{1}, T(\alpha, \beta)\right]$ instead of $I$ satisfy, thanks to $(5.10)$

$$
\|\rho(t)\|_{\mathbf{L}^{\infty}} \leq\left\|\rho^{*}\left(T_{1}\right)\right\|_{\mathbf{L}^{\infty}} \mathrm{e}^{C(\beta)\left(t-T_{1}\right)} \leq\left\|\rho_{0}\right\|_{\mathbf{L}^{\infty}} \mathrm{e}^{C(\beta) T_{1}} \mathrm{e}^{C(\beta)\left(t-T_{1}\right)} \leq\left\|\rho_{0}\right\|_{\mathbf{L}^{\infty}} \mathrm{e}^{C(\beta) T(\alpha, \beta)},
$$

which is less than $\beta$ thanks to the definition of $T(\alpha, \beta)$ and since $\rho_{0} \in \mathcal{X}_{\alpha}$.

Now, we want to define a sequence $\left(T_{n}\right)$ such that $T_{n} \geq T(\alpha, \beta)$ for $n$ sufficiently large. Let assume that the sequence is constructed up to time $T_{n}$ with $T_{n}<T(\alpha, \beta)$. For $\left.\left.T_{n+1} \in\right] T_{n}, T(\alpha, \beta)\right]$, we obtain the contraction estimate

$$
\begin{array}{r}
\left\|\mathcal{Q}\left(\sigma_{1}\right)-\mathcal{Q}\left(\sigma_{2}\right)\right\|_{\mathbf{L}^{\infty}\left(\left[T_{n}, T_{n+1}\right] ; \mathbf{L}^{1}\right)} \leq C\left(T_{n+1}-T_{n}\right) \mathrm{e}^{C^{\prime}\left(T_{n+1}-T_{n}\right)}\left[\mathrm{TV}\left(\rho\left(T_{n}\right)\right)+\left(N W_{N} C\left(T_{n+1}-T_{n}\right)+1\right) \mathrm{e}^{C T_{n}}\left\|\rho_{0}\right\|_{\mathbf{L}^{\infty}}\right] \\
\times\left\|\sigma_{1}-\sigma_{2}\right\|_{\mathbf{L}^{\infty}\left(\left[T_{n}, T_{n+1}\right] ; \mathbf{L}^{1}\right)} \\
\leq\left[\operatorname{TV}\left(\rho_{0}\right) \mathrm{e}^{C^{\prime} T_{n}}+C^{\prime} T_{n} \mathrm{e}^{C^{\prime} T_{n}}+\left(N W_{N} C\left(T_{n+1}-T_{n}\right)+1\right) \mathrm{e}^{C T_{n}}\left\|\rho_{0}\right\|_{\mathbf{L}^{\infty}}\right] \\
\times C^{\prime}\left(T_{n+1}-T_{n}\right) \mathrm{e}^{C^{\prime}\left(T_{n+1}-T_{n}\right)}\left\|\sigma_{1}-\sigma_{2}\right\|_{\mathbf{L}^{\infty}\left(\left[T_{n}, T_{n+1}\right] ; \mathbf{L}^{1}\right)}
\end{array}
$$

where we used the bounds on $\operatorname{TV}\left(\rho\left(T_{n}\right)\right)$ and $\left\|\rho\left(T_{n}\right)\right\|_{\mathbf{L}^{\infty}\left(\mathbb{R}^{N} ; \mathbb{R}\right)}$ provided by Lemma 5.3 and Corollary 5.2 associated to (V1). We may thus extend the solution up to time $T_{n+1}$, where we take $T_{n+1}>T_{n}$ such that

$$
\left[\operatorname{TV}\left(\rho_{0}\right) \mathrm{e}^{C^{\prime} T_{n}}+C T_{n} \mathrm{e}^{C^{\prime} T_{n}}+\left(N W_{N} C\left(T_{n+1}-T_{n}\right)+1\right) \mathrm{e}^{C T_{n}}\left\|\rho_{0}\right\|_{\mathbf{L}^{\infty}}\right] C\left(T_{n+1}-T_{n}\right) \mathrm{e}^{C^{\prime}\left(T_{n+1}-T_{n}\right)}=\frac{1}{2} .
$$

If $T_{n+1}>T(\alpha, \beta)$, then we are done. 
If we assume that the sequence $\left(T_{n}\right)$ defined by induction as above remains less than $T(\alpha, \beta)$, in particular it is bounded. Consequently the left hand side above tends to 0 , whereas the right hand side is taken equal to $1 / 2>0$. Hence, the sequence $\left(T_{n}\right)$ is unbounded. In particular, for $n$ large enough, $T_{n}$ is larger than $T(\alpha, \beta)$; thus the solution to (1.1) is defined on all $I$.

The Lipschitz estimate follows by applying the same procedure as above, in the case when the initial conditions are not the same. The $\mathbf{L}^{\infty}$ and TV bounds follow from (5.10) and from Lemma 5.3.

The proof of Lemma 2.3 directly follows from Lemma 5.3.

Proof of Theorem 2.4. We consider the assumptions (A) and (B) separately.

(A): Let $T>0$, so that $I=[0, T[$, and fix a positive $\alpha$. As in the proof of Theorem 2.2, we define the map

$$
\mathcal{Q}: \begin{aligned}
\mathcal{C}^{0}\left(I ; \mathcal{X}_{\alpha}\right) & \rightarrow \mathcal{C}^{0}\left(I ; \mathcal{X}_{\alpha}\right) \\
\sigma & \mapsto \quad \rho
\end{aligned}
$$

where $\rho$ is the Kružkov solution to (5.15) with $\rho_{0} \in \mathcal{X}_{\alpha}$. The existence of a weak entropy solution for (5.15) in $\mathcal{C}^{\mathbf{0}}\left(I, \mathbf{L}^{\mathbf{1}}\left(\mathbb{R}^{N} ; \mathbb{R}\right)\right)$ is given by Lemma 5.1 , the set of assumptions (V1) allowing to check the hypotheses on $w$ necessary to apply Lemma 5.3. Note that furthermore (A), thanks to Corollary 5.2, gives an $\mathbf{L}^{\infty}$ bound on $\rho$, so that for all $t \in I, \rho(t) \in[0, \alpha]$, a.e. in $x$. Fix $\sigma_{1}, \sigma_{2}$ in $\mathcal{C}^{0}\left(I ; \mathcal{X}_{\alpha}\right)$, call $w_{i}=V\left(\sigma_{i}\right)$ and let $\rho_{1}, \rho_{2}$ be the associated solutions. With the same notations of [15], Theorem 2.6, we let as in the proof of Theorem 2.2,

$$
\kappa_{0}=N W_{N}(2 N+1)\left\|\nabla_{x} w_{1}\right\|_{\mathbf{L}^{\infty}\left(I \times \mathbb{R}^{N} ; \mathbb{R}^{N \times N}\right)}, \quad \kappa=2 N\left\|\nabla_{x} w_{1}\right\|_{\mathbf{L}^{\infty}\left(I \times \mathbb{R}^{N} ; \mathbb{R}^{N \times N}\right)}
$$

so that $\kappa_{0}>\kappa$. Then, by (V1) we have:

$$
\left\|\nabla_{x} V\left(\sigma_{1}\right)\right\|_{\mathbf{L}^{\infty}\left(I \times \mathbb{R}^{N} ; \mathbb{R}^{N \times N}\right)} \leq C\left(\left\|\sigma_{1}\right\|_{\mathbf{L}^{\infty}\left(I \times \mathbb{R}^{N} ; \mathbb{R}^{N \times N}\right)}\right),
$$

and since $\sigma_{1} \in \mathcal{C}^{0}\left(I ; \mathcal{X}_{\alpha}\right)$, we have $\left\|\sigma_{1}\right\|_{\mathbf{L}^{\infty}} \leq \alpha$ so that $\kappa_{0} \leq N W_{N}(2 N+1) C(\alpha)$. Denote

$$
C^{\prime}=N W_{N}(2 N+1) C(\alpha) \quad \text { and } \quad C=C(\alpha) .
$$

The following bounds are also available uniformly for all $\sigma_{1}, \sigma_{2} \in \mathcal{C}^{0}\left(\mathbb{R}_{+} ; \mathcal{X}_{\alpha}\right)$, by $(\mathrm{V} 1)$ :

$$
\begin{aligned}
&\left\|\nabla_{x}^{2} V\left(\sigma_{1}\right)\right\|_{\mathbf{L}^{\infty}\left(I ; \mathbf{L}^{1}\left(\mathbb{R}^{N} ; \mathbb{R}^{N \times N \times N}\right)\right)} \leq C, \\
&\left\|V\left(\sigma_{1}\right)-V\left(\sigma_{2}\right)\right\|_{\mathbf{L}^{\infty}\left(I \times \mathbb{R}^{N} ; \mathbb{R}\right)} \leq C\left\|\sigma_{1}-\sigma_{2}\right\|_{\mathbf{L}^{\infty}\left(I ; \mathbf{L}^{1}\left(\mathbb{R}^{N} ; \mathbb{R}\right)\right)}, \\
&\left\|\operatorname{div}\left(V\left(\sigma_{1}\right)-V\left(\sigma_{2}\right)\right)\right\|_{\mathbf{L}^{\infty}\left(I ; \mathbf{L}^{1}\left(\mathbb{R}^{N} ; \mathbb{R}\right)\right)} \leq C\left\|\sigma_{1}-\sigma_{2}\right\|_{\mathbf{L}^{\infty}\left(I ; \mathbf{L}^{1}\left(\mathbb{R}^{N} ; \mathbb{R}\right)\right)} .
\end{aligned}
$$

Applying [15], Theorem 2.6, we get

$$
\begin{array}{rl}
\left\|\left(\rho_{1}-\rho_{2}\right)(t)\right\|_{\mathbf{L}^{1}} \leq C & C \mathrm{e}^{C^{\prime} t} \operatorname{TV}\left(\rho_{0}\right)\left\|\sigma_{1}-\sigma_{2}\right\|_{\mathbf{L}^{\infty}\left([0, t] ; \mathbf{L}^{1}\right)} \\
& +C^{2} N W_{N} \int_{0}^{t}(t-s) \mathrm{e}^{C^{\prime}(t-s)} \mathrm{d} s\left\|\sigma_{1}-\sigma_{2}\right\|_{\mathbf{L}^{\infty}\left([0, t] ; \mathbf{L}^{1}\right)} \\
& +\int_{0}^{t} C \mathrm{e}^{C^{\prime}(t-s)}\left\|\left(\sigma_{1}-\sigma_{2}\right)(s)\right\|_{\mathbf{L}^{1}} \mathrm{~d} s .
\end{array}
$$

So that

$$
\left\|\mathcal{Q}\left(\sigma_{1}\right)-\mathcal{Q}\left(\sigma_{2}\right)\right\|_{\mathbf{L}^{\infty}\left(I ; \mathbf{L}^{1}\right)} \leq C T \mathrm{e}^{C^{\prime} T}\left[\mathrm{TV}\left(\rho_{0}\right)+N W_{N} C T+1\right]\left\|\sigma_{1}-\sigma_{2}\right\|_{\mathbf{L}^{\infty}\left(I ; \mathbf{L}^{1}\right)} .
$$

Here we introduce the function $f(T)=C T \mathrm{e}^{C^{\prime} T}\left[\mathrm{TV}\left(\rho_{0}\right)+N W_{N} C T+1\right]$ and we remark that $f(T) \rightarrow 0$ when $T \rightarrow 0$. Choose now $T_{1}>0$ so that $f\left(T_{1}\right)=\frac{1}{2}$. Banach Contraction Principle now ensures the existence and uniqueness of a solution to (1.1) on $\left[0, T_{1}\right]$ in the sense of Definition 2.1. 
Let assume that we have defined $T_{1}<T_{2}<\ldots<T_{n}$. Let $T_{n+1}>T_{n}$, then

$$
\begin{array}{r}
\left\|\mathcal{Q}\left(\sigma_{1}\right)-\mathcal{Q}\left(\sigma_{2}\right)\right\|_{\mathbf{L}^{\infty}\left(\left[T_{n}, T_{n+1}\right] ; \mathbf{L}^{1}\right)} \leq C\left(T_{n+1}-T_{n}\right) \mathrm{e}^{C^{\prime}\left(T_{n+1}-T_{n}\right)}\left[\mathrm{TV}\left(\rho\left(T_{n}\right)\right)+N W_{N} C\left(T_{n+1}-T_{n}\right)+1\right] \\
\times\left\|\sigma_{1}-\sigma_{2}\right\|_{\mathbf{L}^{\infty}\left(\left[T_{n}, T_{n+1}\right] ; \mathbf{L}^{1}\right)} \\
\leq\left[\operatorname{TV}\left(\rho_{0}\right) \mathrm{e}^{C^{\prime} T_{n}}+C^{\prime} T_{n} \mathrm{e}^{C^{\prime} T_{n}}+N W_{N} C\left(T_{n+1}-T_{n}\right)+1\right] \\
\times C^{\prime}\left(T_{n+1}-T_{n}\right) \mathrm{e}^{C^{\prime}\left(T_{n+1}-T_{n}\right)}\left\|\sigma_{1}-\sigma_{2}\right\|_{\mathbf{L}^{\infty}\left(\left[T_{n}, T_{n+1}\right] ; \mathbf{L}^{1}\right)}
\end{array}
$$

where we used the bounds on $\left\|\rho\left(T_{n}\right)\right\|_{\mathbf{L}^{\infty}\left(\mathbb{R}^{N} ; \mathbb{R}\right)}$ and $\mathrm{TV}\left(\rho\left(T_{n}\right)\right)$ provided by Corollary 5.2 and Lemma 5.3 associated to the conditions (A) and (V1). We may thus extend the solution up to time $T_{n+1}$, that we define implicitly by

$$
\left[\mathrm{TV}\left(\rho_{0}\right) \mathrm{e}^{C^{\prime} T_{n}}+C T_{n} \mathrm{e}^{C^{\prime} T_{n}}+N W_{N} C\left(T_{n+1}-T_{n}\right)+1\right] C\left(T_{n+1}-T_{n}\right) \mathrm{e}^{C^{\prime}\left(T_{n+1}-T_{n}\right)}=\frac{1}{2} .
$$

Note that this equation defines a unique $T_{n+1}$, the function

$$
T \mapsto\left[\operatorname{TV}\left(\rho_{0}\right) \mathrm{e}^{C^{\prime} T_{n}}+C T_{n} \mathrm{e}^{C^{\prime} T_{n}}+N W_{N} C\left(T-T_{n}\right)+1\right] C\left(T-T_{n}\right) \mathrm{e}^{C^{\prime}\left(T-T_{n}\right)},
$$

being increasing, taking value 0 in $T=T_{n}$ and going to $+\infty$ when $T$ goes to $\infty$.

If the sequence $\left(T_{n}\right)$ is bounded, then the left hand side above tends to 0 , whereas the right hand side is taken equal to $1 / 2>0$. Hence, the sequence $\left(T_{n}\right)$ is unbounded and the solution to (1.1) is defined on all $\mathbb{R}_{+}$.

(S4) follows from Lemma 5.1 and (V1). (S3) is proved as (S1). Note that the Lipschitz constant so obtained is dependent on time. The bound (S2) follows from Lemma 5.3, that gives

$$
\operatorname{TV}(\rho(t)) \leq \operatorname{TV}\left(\rho_{0}\right) \mathrm{e}^{C^{\prime} t}+N W_{N} C t \mathrm{e}^{C^{\prime} t}\left\|\rho_{0}\right\|_{\mathbf{L}^{\infty}} .
$$

(B): Repeat the proof of Theorem 2.2 and, with the notation therein, note that if we find a sequence $\left(\alpha_{n}\right)$ such that $\sum_{n} T\left(\alpha_{n}, \alpha_{n+1}\right)=+\infty$ where $T(\alpha, \beta)=[\ln (\beta / \alpha)] / C(\beta)$, then the solution is defined on the all $\mathbb{R}_{+}$. It is immediate to check that (B) implies that

$$
\sum_{n=1}^{k} T\left(\alpha_{n}, \alpha_{n+1}\right) \geq\left(\|C\|_{\mathbf{L}^{\infty}\left(\mathbb{R}_{+} ; \mathbb{R}_{+}\right)}\right)^{-1} \ln \alpha_{k} \rightarrow+\infty \quad \text { as } k \rightarrow+\infty
$$

completing the proof.

Proof of Proposition 2.5. The bounds on $\rho$ in $\mathbf{W}^{\mathbf{1}, \infty}$ and $\mathbf{W}^{\mathbf{1}, \mathbf{1}}$ follow from Lemma 5.4, thanks to (V2). The $\mathbf{W}^{\mathbf{2}, \mathbf{1}}$ bound comes from (5.14) in Lemma 5.4, thanks to (V3).

\subsection{Gâteaux differentiability}

First of all, if $r_{0} \in\left(\mathbf{L}^{\infty} \cap \mathbf{L}^{\mathbf{1}}\right)\left(\mathbb{R}^{N} ; \mathbb{R}\right)$ and $\rho \in \mathbf{L}^{\infty}\left(I_{\text {ex }} ;\left(\mathbf{W}^{\mathbf{1}, \mathbf{1}} \cap \mathbf{W}^{\mathbf{1}, \infty}\right)\left(\mathbb{R}^{N} ; \mathbb{R}\right)\right)$, we prove that the equation (1.2) admits a unique solution $r \in \mathcal{C}^{\mathbf{0}}\left(I_{\mathrm{ex}} ; \mathbf{L}^{\mathbf{1}}\left(\mathbb{R}^{N} ; \mathbb{R}\right)\right)$.

Proof of Proposition 2.8. We use here once again Lemma 5.1 in order to get an expression of the Kružkov solution for (5.2).

We assume now that $\rho \in \mathcal{C}^{0}\left(I_{\mathrm{ex}} ;\left(\mathbf{W}^{\mathbf{1}, \infty} \cap \mathbf{W}^{\mathbf{1}, \mathbf{1}}\right)\left(\mathbb{R}^{N} ; \mathbb{R}\right)\right)$ and we define $w=V(\rho)$; we also set, for all $s \in \mathcal{C}^{\mathbf{0}}\left(I_{\mathrm{ex}} ; \mathbf{L}^{\mathbf{1}}\left(\mathbb{R}^{N} ; \mathbb{R}\right)\right), R=\operatorname{div}(\rho \mathrm{D} V(\rho)(s))$. Thanks to the assumptions on $\rho$ and $(\mathbf{V} 4)$, we obtain 
$R \in \mathbf{L}^{\infty}\left(I_{\mathrm{ex}} ; \mathbf{L}^{\mathbf{1}}\left(\mathbb{R}^{N} ; \mathbb{R}\right)\right) \cap \mathbf{L}^{\infty}\left(I_{\mathrm{ex}} \times \mathbb{R}^{N} ; \mathbb{R}\right)$. Let $\varepsilon \in \stackrel{\circ}{\mathrm{ex}}_{\mathrm{ex}}$. Then, on $\left[0, T_{\mathrm{ex}}-\varepsilon\right]$ we can apply Lemma 5.1 giving the existence of a Kružkov solution to

$$
\partial_{t} r+\operatorname{div}(r w)=R, \quad r(x, 0)=r_{0} \in\left(\mathbf{L}^{\infty} \cap \mathbf{L}^{\mathbf{1}}\right)\left(\mathbb{R}^{N} ; \mathbb{R}\right) .
$$

Let $T \in\left[0, T_{\text {ex }}-\varepsilon\right]$ and $I=\left[0, T\left[\right.\right.$. We denote $Q$ the application that associates to $s \in \mathcal{C}^{0}\left(I ; \mathbf{L}^{\mathbf{1}}\left(\mathbb{R}^{N} ; \mathbb{R}\right)\right)$ the Kružkov solution $r \in \mathcal{C}^{0}\left(I ; \mathbf{L}^{\mathbf{1}}\left(\mathbb{R}^{N} ; \mathbb{R}\right)\right)$ of $(5.2)$ with initial condition $r_{0} \in\left(\mathbf{L}^{\infty} \cap \mathbf{L}^{1}\right)\left(\mathbb{R}^{N} ; \mathbb{R}\right)$, given by Lemma 5.1. That is to say

$$
\begin{aligned}
Q: s \mapsto r(t, x)= & r_{0}(X(0 ; t, x)) \exp \left(-\int_{0}^{t} \operatorname{div} V(\rho)(\tau, X(\tau ; t, x)) \mathrm{d} \tau\right) \\
& -\int_{0}^{t} \operatorname{div}(\rho \operatorname{DD} V(\rho)(s))(\tau, X(\tau ; t, x)) \exp \left(-\int_{\tau}^{t} \operatorname{div} V(\rho)(u, X(u ; t, x)) \mathrm{d} u\right) \mathrm{d} \tau .
\end{aligned}
$$

Let us give some bounds on $r$. The representation of the solution (5.4) allows indeed to derive a $\mathbf{L}^{\infty}$ bound on $r$. For all $t \in I$, thanks to $(\mathbf{V} 1)$ and $(\mathbf{V} 4)$ we get, with $C=C\left(\|\rho\|_{\mathbf{L}^{\infty}\left(\left[0, T_{\mathrm{ex}}-\varepsilon\right] \times \mathbb{R}^{N} ; \mathbb{R}\right)}\right)$,

$$
\|r(t)\|_{\mathbf{L}^{\infty}} \leq\left\|r_{0}\right\|_{\mathbf{L}^{\infty}} \mathrm{e}^{C t}+t \mathrm{e}^{C t}\|\rho\|_{\mathbf{L}^{\infty}\left([0, t] ; \mathbf{W}^{1, \infty}\right)}\|\mathrm{D} V(\rho)\|_{\mathbf{W}^{1, \infty}}\|s\|_{\mathbf{L}^{\infty}\left([0, t], \mathbf{L}^{1}\right)} .
$$

The same expression allows also to derive a $\mathbf{L}^{\mathbf{1}}$ bound on $r(t)$

$$
\|r(t)\|_{\mathbf{L}^{1}} \leq\left\|r_{0}\right\|_{\mathbf{L}^{1}} \mathrm{e}^{C t}+t \mathrm{e}^{C t}\|\rho\|_{\mathbf{L}^{\infty}\left([0, t] ; \mathbf{W}^{1,1}\right)}\|\mathrm{D} V(\rho)\|_{\mathbf{W}^{1, \infty}}\|s\|_{\mathbf{L}^{\infty}\left([0, t], \mathbf{L}^{1}\right)} .
$$

Now, we want to show that $Q$ is a contraction. We use once again the assumption (V4). For all $s_{1}, s_{2} \in$ $\mathbf{L}^{\infty}\left(I ;\left(\mathbf{L}^{\mathbf{1}} \cap \mathbf{B V}\right)\left(\mathbb{R}^{N} ; \mathbb{R}\right)\right)$ continuous from the right, we have

$$
\left\|\operatorname{div}\left(\rho \mathrm{D} V(\rho)\left(s_{1}-s_{2}\right)\right)\right\|_{\mathbf{L}^{1}\left(\mathbb{R}^{N} ; \mathbb{R}\right)} \leq C\|\rho\|_{\mathbf{W}^{1,1}\left(\mathbb{R}^{N} ; \mathbb{R}\right)}\left\|s_{1}-s_{2}\right\|_{\mathbf{L}^{1}\left(\mathbb{R}^{N} ; \mathbb{R}\right)} .
$$

Thus, we get:

$$
\begin{aligned}
\left\|Q\left(s_{1}\right)-Q\left(s_{2}\right)\right\|_{\mathbf{L}^{\infty}\left(I ; \mathbf{L}^{1}\right)} & \leq C\|\rho\|_{\mathbf{L}^{\infty}\left(I ; \mathbf{W}^{1,1}\right)}\left\|s_{1}-s_{2}\right\|_{\mathbf{L}^{\infty}\left(I ; \mathbf{L}^{1}\right)} \int_{0}^{T} \exp \left((T-\tau)\|\operatorname{div} V(\rho)\|_{\mathbf{L}^{\infty}}\right) \mathrm{d} \tau \\
& \leq\left(\mathrm{e}^{C T}-1\right)\|\rho\|_{\mathbf{L}^{\infty}\left(\left[0, T_{\mathrm{ex}}-\varepsilon\right] ; \mathbf{W}^{1,1}\right)}\left\|s_{1}-s_{2}\right\|_{\mathbf{L}^{\infty}\left(I ; \mathbf{L}^{1}\right)} .
\end{aligned}
$$

Then, for $T$ small enough, can apply the Fixed Point Theorem, that gives us the existence of a unique Kružkov solution to the problem. Furthermore, as the time of existence does not depend on the initial condition, we can iterate this procedure to obtain existence on the interval $\left[0, T_{\mathrm{ex}}-\varepsilon\right]$. Finally, as this is true for all $\varepsilon \in \check{I}_{\mathrm{ex}}$, we obtain the same result on the all interval $I_{\mathrm{ex}}$.

The $\mathbf{L}^{1}$ bound follows from (5.4). Let $T \in I_{\text {ex }}$ and $t \in I$, then for a suitable $C=C\left(\|\rho\|_{\mathbf{L}^{\infty}\left(I \times \mathbb{R}^{N} ; \mathbb{R}\right)}\right)$

$$
\|r(t)\|_{\mathbf{L}^{1}} \leq\left\|r_{0}\right\|_{\mathbf{L}^{1}} \mathrm{e}^{C t}+\|\rho\|_{\mathbf{L}^{\infty}\left(I ; \mathbf{W}^{1,1}\right)}\|\operatorname{div} \mathrm{D} V(\rho)\|_{\mathbf{L}^{\infty}} \int_{0}^{t}\|r(\tau)\|_{\mathbf{L}^{1}} \mathrm{~d} \tau .
$$

A use of (V4) and an application of Gronwall Lemma gives

$$
\|r(t)\|_{\mathbf{L}^{1}} \leq \mathrm{e}^{C t} \mathrm{e}^{K\|\rho\|_{\mathbf{L}^{\infty}\left(I, \mathbf{w}^{\mathbf{1}, \mathbf{1}}\right)^{t}}\left\|r_{0}\right\|_{\mathbf{L}^{1}},}
$$

where $K=K\left(\|\rho\|_{\mathbf{L}^{\infty}\left(I \times \mathbb{R}^{N} ; \mathbb{R}\right)}\right)$ is as in (V4). 
The $\mathbf{L}^{\infty}$ bound comes from the same representation formula. Indeed, for $T \in I_{\mathrm{ex}}$ and $t \in I$ we have

$$
\|r(t)\|_{\mathbf{L}^{\infty}} \leq \mathrm{e}^{C t}\left\|r_{0}\right\|_{\mathbf{L}^{\infty}}+\|\rho\|_{\mathbf{L}^{\infty}\left(I ; \mathbf{W}^{1, \infty}\right)}\|\operatorname{div} \mathrm{D} V(\rho)\|_{\mathbf{L}^{\infty}} \int_{0}^{t}\|r(\tau)\|_{\mathbf{L}^{1}} \mathrm{~d} \tau .
$$

Then, the last $\|r(\tau)\|_{\mathbf{L}^{1}}$ is bounded just as above. We get

$$
\|r(t)\|_{\mathbf{L}^{\infty}} \leq \mathrm{e}^{C t}\left\|r_{0}\right\|_{\mathbf{L}^{\infty}}+K t \mathrm{e}^{2 C t} \mathrm{e}^{K\|\rho\|_{\mathbf{L}^{\infty}\left(I, \mathbf{W}^{1,1}\right)}}\left\|r_{0}\right\|_{\mathbf{L}^{1}}\|\rho\|_{\mathbf{L}^{\infty}\left(I, \mathbf{W}^{1, \infty}\right)} .
$$

Finally, we get a $\mathbf{W}^{\mathbf{1 , 1}}$ bound using the expression of the solution given by Lemma 5.1. Indeed, assuming in addition (V2) and (V4), we get

$$
\begin{aligned}
\|\nabla r(t)\|_{\mathbf{L}^{1}\left(\mathbb{R}^{N} ; \mathbb{R}\right)} \leq & \mathrm{e}^{2 C t}\left\|\nabla r_{0}\right\|_{\mathbf{L}^{1}}+C t \mathrm{e}^{2 C t}\left\|r_{0}\right\|_{\mathbf{L}^{1}}+K(1+C t) \mathrm{e}^{2 C t}\|\rho\|_{\mathbf{L}^{\infty}\left(I ; \mathbf{W}^{2,1}\right)} \int_{0}^{t}\|r(\tau)\|_{\mathbf{L}^{1}} \mathrm{~d} \tau \\
\leq & \mathrm{e}^{2 C t}\left\|\nabla r_{0}\right\|_{\mathbf{L}^{1}}+C t \mathrm{e}^{2 C t}\left\|r_{0}\right\|_{\mathbf{L}^{1}} \\
& +K t(1+C t) \mathrm{e}^{3 C t} \mathrm{e}^{K\|\rho\|_{\mathbf{L}^{\infty}\left(I, \mathbf{W}^{1,1}\right)}}\left\|r_{0}\right\|_{\mathbf{L}^{1}}\|\rho\|_{\mathbf{L}^{\infty}\left(I ; \mathbf{W}^{2,1}\right)} .
\end{aligned}
$$

Hence, denoting $C^{\prime}=\max \left\{C, K\|\rho\|_{\mathbf{L}^{\infty}\left(I, \mathbf{W}^{1,1}\right)}\right\}$, we obtain

$$
\|r(t)\|_{\mathbf{W}^{1,1}} \leq\left\|r_{0}\right\|_{\mathbf{W}^{1,1}}\left(1+C^{\prime} t\right) \mathrm{e}^{2 C^{\prime} t}+K t(1+C t) \mathrm{e}^{4 C^{\prime} t}\left\|r_{0}\right\|_{\mathbf{L}^{1}}\|\rho\|_{\mathbf{L}^{\infty}\left(I ; \mathbf{W}^{2,1}\right)}
$$

concluding the proof.

Now, we address the weak Gâteaux differentiability of the semigroup generated by (1.1).

Proof of Proposition 2.9. Let $\alpha=\max \left\{\left\|\rho_{0}\right\|_{\mathbf{L}^{\infty}}, 1\right\}$ and $\beta>\alpha$. Fix $h \in\left[0, h^{*}\right]$ with $h^{*}$ small enough so that $\beta>\alpha\left(1+h^{*}\right)$. Note that $\rho_{0}, r_{0} \in \mathcal{X}_{\alpha}$. By Theorem 2.2, (1.1) admits the weak entropy solutions $\rho \in \mathcal{C}^{0}\left([0, T(\alpha, \beta)] ; \mathcal{X}_{\beta}\right)$, and $(2.3)$ admits the solution $\rho_{h} \in \mathcal{C}^{\mathbf{0}}\left([0, T(\alpha(1+h), \beta)] ; \mathcal{X}_{\beta}\right)$. Note that

$$
T(\alpha(1+h), \beta)=\frac{\ln (\beta /(\alpha(1+h)))}{C(\beta)}=T(\alpha, \beta)-\frac{\ln (1+h)}{C(\beta)} \leq T(\alpha, \beta)
$$

and $T(\alpha(1+h), \beta)$ tends to $T(\alpha, \beta)$ as $h$ goes to 0 . In particular, both solutions are defined on the interval $\left[0, T_{*}\right]$, where $T_{*}=T\left(\alpha\left(1+h^{*}\right), \beta\right)$.

Write now the definition of weak solution for $\rho, \rho_{h}$. Let $\varphi \in \mathcal{C}_{c}^{\infty}\left(\left[0, T_{*}\right] \times \mathbb{R}^{N} ; \mathbb{R}\right)$

$$
\begin{array}{r}
\int_{\mathbb{R}_{+}^{*}} \int_{\mathbb{R}^{N}}\left(\rho \partial_{t} \varphi+(\rho V(\rho)) \cdot \nabla_{x} \varphi\right) \mathrm{d} x \mathrm{~d} t=0, \\
\int_{\mathbb{R}_{+}^{*}} \int_{\mathbb{R}^{N}}\left(\rho_{h} \partial_{t} \varphi+\left(\rho_{h} V\left(\rho_{h}\right)\right) \cdot \nabla_{x} \varphi\right) \mathrm{d} x \mathrm{~d} t=0
\end{array}
$$

use (V4) and write, for a suitable function $\varepsilon=\varepsilon\left(\rho, \rho_{h}\right), V\left(\rho_{h}\right)=V(\rho)+\mathrm{D} V(\rho)\left(\rho_{h}-\rho\right)+\varepsilon\left(\rho, \rho_{h}\right)$, with $\left\|\varepsilon\left(\rho, \rho_{h}\right)\right\|_{\mathbf{L}^{\infty}\left(\mathbb{R}^{N} ; \mathbb{R}\right)} \leq K(2 \beta)\left(\left\|\rho_{h}-\rho\right\|_{\mathbf{L}^{1}\left(\mathbb{R}^{N} ; \mathbb{R}\right)}\right)^{2}$. Then,

$$
\rho V(\rho)-\rho_{h} V\left(\rho_{h}\right)=\left(\rho-\rho_{h}\right) V(\rho)+\rho \mathrm{D} V(\rho)\left(\rho-\rho_{h}\right)+\left(\rho-\rho_{h}\right) \mathrm{D} V(\rho)\left(\rho-\rho_{h}\right)-\rho_{h} \varepsilon\left(\rho, \rho_{h}\right) .
$$


Consequently,

$$
\begin{aligned}
\int_{\mathbb{R}_{+}^{*}} \int_{\mathbb{R}^{N}}\left[\frac{\rho-\rho_{h}}{h} \partial_{t} \varphi+\left(\frac{\rho-\rho_{h}}{h} V(\rho)+\rho \mathrm{D} V(\rho)\left(\frac{\rho-\rho_{h}}{h}\right)\right.\right. & \left.\left.+\frac{\rho-\rho_{h}}{h} \mathrm{D} V(\rho)\left(\rho-\rho_{h}\right)-\rho_{h} \frac{\varepsilon\left(\rho, \rho_{h}\right)}{h}\right) \cdot \nabla_{x} \varphi\right] \mathrm{d} x \mathrm{~d} t=0 .
\end{aligned}
$$

Using (V4), $\rho(t) \in \mathcal{X}_{\beta}$ and the estimate on $\varepsilon$ we obtain for all $t \in\left[0, T_{*}\right]$ :

$$
\begin{aligned}
& \int_{\mathbb{R}^{N}}\left|\nabla_{x} \varphi\right| \mid \frac{\rho-\rho_{h}}{h} \mathrm{DV}(\rho)\left(\rho-\rho_{h}\right)-\rho_{h} \frac{\varepsilon\left(\rho, \rho_{h}\right)}{h} \mid \mathrm{d} x \\
& \leq \int_{\mathbb{R}^{N}}\left(C(\beta)\left|\rho-\rho_{h}\right|+\beta K(2 \beta)\left\|\rho-\rho_{h}\right\|_{\mathbf{L}^{1}}\right)\left\|\frac{\rho-\rho_{h}}{h}\right\|_{\mathbf{L}^{1}}\left|\nabla_{x} \varphi\right| \mathrm{d} x
\end{aligned}
$$$$
\rightarrow 0 \quad \text { as } \quad h \rightarrow 0
$$

by the Dominated Convergence Theorem, since $\frac{\rho_{h}-\rho}{h}$ is bounded in $\mathbf{L}^{\mathbf{1}}\left(\left[0, T_{*}\right] \times \mathbb{R}^{N} ; \mathbb{R}\right)$. Then, if $\left(\rho_{h}-\rho\right) / h^{h \rightarrow 0} r$, we get

$$
\int_{\mathbb{R}} \int_{\mathbb{R}^{N}}\left[r \partial_{t} \varphi+(r V(\rho)+\rho \mathrm{D} V(\rho)(r)) \cdot \nabla_{x} \varphi\right] \mathrm{d} x \mathrm{~d} t=0
$$

That is to say, $r$ is a distributional solution to (1.2).

As this is true for all $h^{*}$ small enough, finally we obtain a solution on the all interval $[0, T(\alpha, \beta)[$. Hence, we observe that if $\rho \in \mathcal{C}^{0}\left(I_{\text {ex }} \times \mathbb{R}^{N} ; \mathbb{R}\right)$, then $r$ is defined on all $I_{\text {ex }}$.

Assume now that (V4) and (V5) are satisfied by $V$. We want to show that with these hypotheses, we have now strong convergence in $\mathbf{L}^{\mathbf{1}}$ to the Kružkov solution of (1.2).

Proof of Theorem 2.10. Let $\alpha, \beta>0$ with $\beta>\alpha$, and $h \in\left[0, h^{*}\right]$ with $h^{*}$ small enough so that $\beta>\alpha\left(1+h^{*}\right)$. Let us denote $T(h)=T(\alpha(1+h), \beta)$ for $h \in\left[0, h^{*}\right]$ the time of existence of the solution of $(1.1)$ given by Theorem 2.2 .

Fix $\rho_{0} \in\left(\mathbf{W}^{\mathbf{1}, \infty} \cap \mathbf{W}^{\mathbf{2}, \mathbf{1}}\right)\left(\mathbb{R}^{N} ;[0, \alpha]\right), r_{0} \in\left(\mathbf{L}^{\infty} \cap \mathbf{W}^{\mathbf{1}, \mathbf{1}}\right)\left(\mathbb{R}^{N} ;[0, \alpha]\right)$. Let $\rho$, respectively $\rho_{h}$, be the weak entropy solutions of (1.1) given by Theorem 2.2 with initial condition $\rho_{0}$, respectively $\rho_{0}+h r_{0}$. Note that these both solutions are in $\mathcal{C}^{0}\left(\left[0, T\left(h^{*}\right)\right] ; \mathbf{L}^{\mathbf{1}}\left(\mathbb{R}^{N} ; \mathbb{R}\right)\right)$. Furthermore, under these hypotheses for $\rho_{0}$ and $r_{0}$, we get thanks to Proposition 2.5 that the corresponding solutions $\rho$ and $\rho_{h}$ of $(1.1)$ are in $\mathcal{C}^{\mathbf{0}}\left(\left[0, T\left(h^{*}\right)\right] ;\left(\mathbf{W}^{\mathbf{1}, \infty} \cap\right.\right.$ $\left.\left.\mathbf{W}^{\mathbf{2}, \mathbf{1}}\right)\left(\mathbb{R}^{N} ;[0, \beta]\right)\right)$, condition (V3) being satisfied. Hence, we can now introduce the Kružkov solution $r \in$ $\mathcal{C}^{\mathbf{0}}\left(\left[0, T\left(h^{*}\right)\left[; \mathbf{L}^{\mathbf{1}}\left(\mathbb{R}^{N} ; \mathbb{R}\right)\right)\right.\right.$ of $(1.2)$, whose existence is given in this case by Proposition 2.8. Note that, $r_{0}$ being in $\mathbf{W}^{\mathbf{1}, \mathbf{1}}\left(\mathbb{R}^{N} ; \mathbb{R}\right)$ and $\rho \in \mathbf{L}^{\infty}\left(\left[0, T\left(h^{*}\right)\right] ; \mathbf{W}^{\mathbf{2}, \mathbf{1}}\left(\mathbb{R}^{N} ; \mathbb{R}\right)\right)$ and (V2), (V4) being satisfied, $r(t)$ is also in $\mathbf{W}^{\mathbf{1}, \mathbf{1}}\left(\mathbb{R}^{N} ; \mathbb{R}\right)$ for all $t \in\left[0, T\left(h^{*}\right)\left[\right.\right.$ thanks to the $\mathbf{W}^{\mathbf{1}, \mathbf{1}}$ bound of Proposition 2.8 .

Let us denote $z_{h}=\rho+h r$. We would like to compare $\rho_{h}$ and $z_{h}$ thanks to [15], Theorem 2.6. A straightforward computation shows that $z_{h}$ is the solution to the following problem,

$$
\left\{\begin{array}{l}
\partial_{t} z_{h}+\operatorname{div}\left(z_{h}(V(\rho)+h \mathrm{D} V(\rho)(r))\right)=h^{2} \operatorname{div}(r \mathrm{D} V(\rho)(r)) \\
z_{h}(0)=\rho_{0}+h r_{0} \in \mathcal{X}_{\alpha(1+h)} .
\end{array}\right.
$$

Note that the source term being in $\mathcal{C}^{\mathbf{0}}\left(\left[0, T\left(h^{*}\right)\left[; \mathbf{L}^{\mathbf{1}}\left(\mathbb{R}^{N} ; \mathbb{R}\right)\right)\right.\right.$, and the flow being regular, we can apply to this equation Lemma 5.1 that gives existence of a Kružkov solution.

As in the proof of Lemma 5.1, we make here the remark that [15], Theorem 2.6, can be used with the second source term in $\mathcal{C}^{\mathbf{0}}\left(\left[0, T\left(h^{*}\right)\left[; \mathbf{L}^{\mathbf{1}}\left(\mathbb{R}^{N} ; \mathbb{R}\right)\right)\right.\right.$ and the flow $\mathcal{C}^{\mathbf{2}}$ in space and only $\mathcal{C}^{\mathbf{0}}$ in time. Besides, we 
also use the same slight improvement as in the proof of Lemma 5.3, taking the $\mathbf{L}^{\infty}$ norm in the integral term only in space, keeping the time fixed. We get, with $\kappa_{0}=N W_{N}(2 N+1)\left\|\nabla_{x} V\left(\rho_{h}\right)\right\|_{\mathbf{L}^{\infty}\left(\left[0, T\left(h^{*}\right)\right] \times \mathbb{R}^{N ;} ; \mathbb{R}\right)}$ and $\kappa=2 N\left\|\nabla_{x} V\left(\rho_{h}\right)\right\|_{\mathbf{L}^{\infty}\left(\left[0, T\left(h^{*}\right)\right] \times \mathbb{R}^{N} ; \mathbb{R}\right)}$, for some $T \in\left[0, T\left(h^{*}\right)\right]$ and with $I=[0, T]$,

$$
\begin{aligned}
\left\|\rho_{h}-z_{h}\right\|_{\mathbf{L}^{\infty}\left(I ; \mathbf{L}^{1}\right)} \leq & T \mathrm{e}^{\kappa_{0} T} \operatorname{TV}\left(\rho_{0}+h r_{0}\right)\left\|V\left(\rho_{h}\right)-V(\rho)-h \mathrm{D} V(\rho)(r)\right\|_{\mathbf{L}^{\infty}\left(\left[0, T\left(h^{*}\right)\right] \times \mathbb{R}^{N} ; \mathbb{R}^{N}\right)} \\
& +N W_{N} \int_{0}^{T}(T-t) \mathrm{e}^{\kappa_{0}(T-t)} \int_{\mathbb{R}^{N}}\left\|\rho_{h}(t)\right\|_{\mathbf{L}^{\infty}}\left\|\nabla_{x} \operatorname{div} V\left(\rho_{h}\right)\right\| \mathrm{d} x \mathrm{~d} t \\
& \times\left\|V\left(\rho_{h}\right)-V(\rho)-h \mathrm{D} V(\rho)(r)\right\|_{\mathbf{L}^{\infty}\left(\left[0, T\left(h^{*}\right)\right] \times \mathbb{R}^{N} ; \mathbb{R}^{N}\right)} \\
& +h^{2} \int_{0}^{T} \mathrm{e}^{\kappa(T-t)} \int_{\mathbb{R}^{N}}|\operatorname{div}(r \mathrm{D} V(\rho)(r))| \mathrm{d} x \mathrm{~d} t \\
& +\int_{0}^{T} \mathrm{e}^{\kappa(T-t)} \int_{\mathbb{R}^{N}}\left|\operatorname{div}\left(V\left(\rho_{h}\right)-V(\rho)-h \mathrm{D} V(\rho)(r)\right)\right| \mathrm{d} x \mathrm{~d} t \\
& \times \max _{t \in[0, T]}\left\{\left\|\rho_{h}(t)\right\|_{\mathbf{L}^{\infty}},\left\|z_{h}(t)\right\|_{\mathbf{L}^{\infty}}\right\} .
\end{aligned}
$$

Then, setting $C=C(\beta)$ and $K=K(2 \beta)$, we use:

- the bound of $\rho$ and $\rho_{h}$ in $\mathbf{L}^{\infty}$ given by Corollary 5.2

$$
\|\rho(t)\|_{\mathbf{L}^{\infty}} \leq\left\|\rho_{0}\right\|_{\mathbf{L}^{\infty}} \mathrm{e}^{C t} \leq \beta \quad \text { and } \quad\left\|\rho_{h}(t)\right\|_{\mathbf{L}^{\infty}} \leq\left\|\rho_{0}+h r_{0}\right\|_{\mathbf{L}^{\infty}} \mathrm{e}^{C t} \leq \beta ;
$$

- the properties of $V$ given in (V1) to get

$$
\left\|\nabla_{x} \operatorname{div} V\left(\rho_{h}\right)\right\|_{\mathbf{L}^{\infty}\left(\mathbb{R}_{+} \times \mathbb{R}^{N} ; \mathbb{R}\right)} \leq C \quad \text { and } \quad\left\|\nabla_{x} \operatorname{div} V\left(\rho_{h}\right)\right\|_{\mathbf{L}^{\infty}\left(I ; \mathbf{L}^{1}\left(\mathbb{R}^{N} ; \mathbb{R}\right)\right)} \leq C
$$

- the property (V4), respectively (V5), to get

$$
\begin{gathered}
\left\|V\left(\rho_{h}\right)-V(\rho)-h \mathrm{D} V(\rho)(r)\right\|_{\mathbf{L}^{\infty}\left(I \times \mathbb{R}^{N} ; \mathbb{R}\right)} \leq K\left(\left\|\rho_{h}-\rho\right\|_{\mathbf{L}^{\infty}\left(I ; \mathbf{L}^{1}\left(\mathbb{R}^{N} ; \mathbb{R}\right)\right)}^{2}+\left\|\rho_{h}-z_{h}\right\|_{\mathbf{L}^{\infty}\left(I ; \mathbf{L}^{1}\left(\mathbb{R}^{N} ; \mathbb{R}\right)\right)}\right), \text { respectively } \\
\left\|\operatorname{div}\left(V\left(\rho_{h}\right)-V(\rho)-h \mathrm{D} V(\rho)(r)\right)\right\|_{\mathbf{L}^{\infty}\left(I ; \mathbf{L}^{1}\left(\mathbb{R}^{N} ; \mathbb{R}\right)\right)} \leq K\left(\left\|\rho_{h}-\rho\right\|_{\mathbf{L}^{\infty}\left(I ; \mathbf{L}^{1}\left(\mathbb{R}^{N} ; \mathbb{R}\right)\right)}^{2}+\left\|\rho_{h}-z_{h}\right\|_{\mathbf{L}^{\infty}\left(I ; \mathbf{L}^{1}\left(\mathbb{R}^{N} ; \mathbb{R}\right)\right)}\right)
\end{gathered}
$$

- the property (V4) to get

$$
\|\operatorname{div}(r \mathrm{D} V(\rho)(r))\|_{\mathbf{L}^{1}} \leq K\|r\|_{\mathbf{W}^{1,1}}\|r\|_{\mathbf{L}^{1}} .
$$

Gathering all these estimates, denoting $C^{\prime}=N W_{N}(2 N+1) C$, we obtain

$$
\begin{aligned}
\left\|\rho_{h}-z_{h}\right\|_{\mathbf{L}^{\infty}\left(I ; \mathbf{L}^{1}\right)} \leq & T \mathrm{e}^{C^{\prime} T}\left(\operatorname{TV}\left(\rho_{0}+h r_{0}\right)+N W_{N} C T \beta\right) K\left(\left\|\rho_{h}-\rho\right\|_{\mathbf{L}^{\infty}\left(I ; \mathbf{L}^{1}\right)}^{2}+\left\|\rho_{h}-z_{h}\right\|_{\mathbf{L}^{\infty}\left(I ; \mathbf{L}^{1}\right)}\right) \\
& +h^{2} K T \mathrm{e}^{C^{\prime} T}\|r\|_{\mathbf{L}^{\infty}\left(I ; \mathbf{W}^{1,1}\right)}\|r\|_{\mathbf{L}^{\infty}\left(I ; \mathbf{L}^{1}\right)} \\
& +\left(\beta+h \sup _{t \in I}\|r(t)\|_{\mathbf{L}^{\infty}}\right) T \mathrm{e}^{C^{\prime} T} K\left(\left\|\rho_{h}-\rho\right\|_{\mathbf{L}^{\infty}\left(I ; \mathbf{L}^{1}\right)}^{2}+\left\|\rho_{h}-z_{h}\right\|_{\mathbf{L}^{\infty}\left(I ; \mathbf{L}^{1}\right)}\right) .
\end{aligned}
$$

Then, dividing by $h$ and introducing

$$
F_{h}(T)=K T \mathrm{e}^{C^{\prime} T}\left[\mathrm{TV}\left(\rho_{0}\right)+h \mathrm{TV}\left(r_{0}\right)+N W_{N} C T \beta+\beta+h\|r(t)\|_{\mathbf{L}^{\infty}}\right],
$$


we obtain

$$
\begin{aligned}
\left\|\frac{\rho_{h}-z_{h}}{h}\right\|_{\mathbf{L}^{\infty}\left(I ; \mathbf{L}^{1}\right)} \leq & F_{h}(T)\left[\left\|\rho_{h}-\rho\right\|_{\mathbf{L}^{\infty}\left(I ; \mathbf{L}^{1}\right)}\left\|\frac{\rho_{h}-\rho}{h}\right\|_{\mathbf{L}^{\infty}\left(I ; \mathbf{L}^{1}\right)}+\left\|\frac{\rho_{h}-z_{h}}{h}\right\|_{\mathbf{L}^{\infty}\left(I ; \mathbf{L}^{1}\right)}\right] \\
& +h K T \mathrm{e}^{C^{\prime} T}\|r\|_{\mathbf{L}^{\infty}\left(I ; \mathbf{W}^{1,1}\right)}\|r\|_{\mathbf{L}^{\infty}\left(I ; \mathbf{L}^{1}\right)} .
\end{aligned}
$$

Note that $F_{h}$ is a function that vanishes in $T=0$ and that depends also on $\rho_{0}, r_{0}$ and $h$. Hence, we can find $\bar{T} \leq T\left(h^{*}\right)$ small enough such that $F_{h^{*}}(\bar{T}) \leq 1 / 2$. Furthermore, for all $T \leq T\left(h^{*}\right), h \mapsto F_{h}(T)$ is increasing and hence $h \leq h^{*}$ implies $F_{h}(T) \leq F_{h^{*}}(T)$. Noting moreover that $\left\|\frac{\rho_{h}-\rho}{h}\right\|_{\mathbf{L}^{\infty}\left(I ; \mathbf{L}^{1}\right)}$ has a uniform bound $M$ in $h$ by (2) in Theorem 2.2, we get for $T \leq \bar{T}$

$$
\begin{aligned}
\frac{1}{2}\left\|\frac{\rho_{h}-\rho}{h}-r\right\|_{\mathbf{L}^{\infty}\left(I ; \mathbf{L}^{1}\right)} & =\frac{1}{2}\left\|\frac{\rho_{h}-z_{h}}{h}\right\|_{\mathbf{L}^{\infty}\left(I ; \mathbf{L}^{1}\right)} \\
& \leq \frac{M}{2}\left\|\rho_{h}-\rho\right\|_{\mathbf{L}^{\infty}\left(I ; \mathbf{L}^{1}\right)}+h K T \mathrm{e}^{C^{\prime} T}\|r\|_{\mathbf{L}^{\infty}\left(I, \mathbf{W}^{1,1}\right)}\|r\|_{\mathbf{L}^{\infty}\left(I ; \mathbf{L}^{1}\right)} .
\end{aligned}
$$

The right side above goes to 0 when $h \rightarrow 0$, so we have proved the Gâteaux differentiability of the semigroup $S$ for small time. Finally, we iterate like in the proof of Theorem 2.2 in order to have existence on the all interval $\left[0, T\left(h^{*}\right)\right]$. Let $T_{1}$ be such that $F_{h^{*}}\left(T_{1}\right)=1 / 2$ and assume $T_{1}<T\left(h^{*}\right)$. If we assume the Gâteaux differentiability is proved until time $T_{n} \leq T\left(h^{*}\right)$, we make the same estimate on $\left[T_{n}, T_{n+1}\right], T_{n+1}$ being to determine. We get

$$
\begin{aligned}
\left\|\rho_{h}-z_{h}\right\|_{\mathbf{L}^{\infty}\left(\left[T_{n}, T_{n+1}\right] ; \mathbf{L}^{1}\right)} \leq & \left(T_{n+1}-T_{n}\right) \mathrm{e}^{C^{\prime}\left(T_{n+1}-T_{n}\right)}\left(\mathrm{TV}\left(\rho_{h}\left(T_{n}\right)\right)+N W_{N} C\left(T_{n+1}-T_{n}\right) \beta\right) \\
& \times K\left(\left\|\rho_{h}-\rho\right\|_{\mathbf{L}^{\infty}\left(\left[T_{n}, T_{n+1}\right] ; \mathbf{L}^{1}\right)}^{2}+\left\|\rho_{h}-z_{h}\right\|_{\mathbf{L}^{\infty}\left(\left[T_{n}, T_{n+1}\right] ; \mathbf{L}^{1}\right)}\right) \\
& +h^{2} K\left(T_{n+1}-T_{n}\right) \mathrm{e}^{C^{\prime}\left(T_{n+1}-T_{n}\right)}\|r\|_{\mathbf{L}^{\infty}\left(\left[T_{n}, T_{n+1}\right] ; \mathbf{W}^{1,1}\right)}\|r\|_{\mathbf{L}^{\infty}\left(\left[T_{n}, T_{n+1}\right] ; \mathbf{L}^{1}\right)} \\
& +\left(\beta+h \sup _{\left[T_{n}, T_{n+1}\right]}\|r(t)\|_{\mathbf{L}^{\infty}}\right)\left(T_{n+1}-T_{n}\right) \mathrm{e}^{C^{\prime}\left(T_{n+1}-T_{n}\right)} \\
& \times K\left(\left\|\rho_{h}-\rho\right\|_{\mathbf{L}^{\infty}\left(\left[T_{n}, T_{n+1}\right] ; \mathbf{L}^{1}\right)}^{2}+\left\|\rho_{h}-z_{h}\right\|_{\mathbf{L}^{\infty}\left(\left[T_{n}, T_{n+1}\right] ; \mathbf{L}^{1}\right)}\right)
\end{aligned}
$$

Then, we divide by $h$ and we introduce, for $T \geq T_{n}$

$$
\begin{aligned}
F_{h, n}(T)= & K\left(T-T_{n}\right) \mathrm{e}^{C^{\prime}\left(T-T_{n}\right)}\left[\left(\mathrm{TV}\left(\rho_{0}\right)+h \mathrm{TV}\left(r_{0}\right)\right) \mathrm{e}^{C T_{n}}+\beta C^{\prime} T_{n} \mathrm{e}^{C^{\prime} T_{n}}\right. \\
& \left.+N W_{N} C\left(T-T_{n}\right) \beta+\beta+h \sup _{\left[T_{n}, T_{n+1}\right]}\|r(t)\|_{\mathbf{L} \infty}\right] .
\end{aligned}
$$

We define $T_{n+1}>T_{n}$ such that $F_{h, n}\left(T_{n+1}\right)=\frac{1}{2}$. This is possible since $F_{h, n}$ vanishes in $T=T_{n}$ and increases to infinity when $T \rightarrow \infty$. Hence, as long as $T_{n+1} \leq T\left(h^{*}\right)$, we get

$$
\begin{aligned}
\left\|\frac{\rho_{h}-\rho}{h}-r\right\|_{\mathbf{L}^{\infty}\left(\left[T_{n}, T_{n+1}\right] ; \mathbf{L}^{1}\right)} \leq & K M\left\|\rho_{h}-\rho\right\|_{\mathbf{L}^{\infty}\left(\left[T_{n}, T_{n+1}\right] ; \mathbf{L}^{1}\right)} \\
& +2 h K\left(T_{n+1}-T_{n}\right) \mathrm{e}^{C^{\prime}\left(T_{n+1}-T_{n}\right)}\|r\|_{\mathbf{L}^{\infty}\left(\left[T_{n}, T_{n+1}\right], \mathbf{W}^{1,1}\right)}\|r\|_{\mathbf{L}^{\infty}\left(\left[T_{n}, T_{n+1}\right] ; \mathbf{L}^{1}\right)} .
\end{aligned}
$$

The next question is to wonder if $\left(T_{n}\right)$ goes up to $T\left(h^{*}\right)$. We assume that it is not the case: then necessarily, $F_{h, n}\left(T_{n+1}\right) \stackrel{n \rightarrow \infty}{\longrightarrow} 0$, since $T_{n+1}-T_{n} \rightarrow 0$. This is a contradiction to $F_{h, n}\left(T_{n+1}\right)=1 / 2$. 
Consequently, $T_{n} \stackrel{n \rightarrow \infty}{\longrightarrow} \infty$ and the Gâteaux differentiability is valid for all time $t \in\left[0, T\left(h^{*}\right)\right]$. Then, making $h^{*}$ goes to 0 , we obtain that the differentiability is valid on in the interval $[0, T(\alpha, \beta)[$.

It remains to check that the Gâteaux derivative is a bounded linear operator, for $t$ and $\rho_{0}$ fixed. The linearity is immediate. Additionally, due to the $\mathbf{L}^{1}$ estimate on the solution $r$ of the linearized equation (1.2) given by Proposition 2.8, we obtain

$$
\left\|\mathrm{D} S_{t}\left(\rho_{0}\right)\left(r_{0}\right)\right\|_{\mathbf{L}^{1}}=\|r(t)\|_{\mathbf{L}^{1}} \leq \mathrm{e}^{K t\|\rho\|_{\mathbf{L}^{\infty}\left(I ; \mathbf{W}^{1,1}\right)}} \mathrm{e}^{C t}\left\|r_{0}\right\|_{\mathbf{L}^{1}},
$$

so that the Gâteaux derivative is bounded, at least for $t \leq T<T_{\mathrm{ex}}$.

\subsection{Proofs related to Sections 3 and 4}

Proof of Proposition 3.1. Note that $v(\rho)$ is constant in $x$, hence $\operatorname{div} V(\rho)=0$, and (A) is satisfied. Besides, we easily obtain $\left\|\partial_{x} V(\rho)\right\|_{\mathbf{L}^{\infty}(\mathbb{R} ; \mathbb{R})}=0,\left\|\partial_{x} V(\rho)\right\|_{\mathbf{L}^{1}(\mathbb{R} ; \mathbb{R})}=0,\left\|\partial_{x}^{2} V(\rho)\right\|_{\mathbf{L}^{1}(\mathbb{R} ; \mathbb{R})}=0$ and

$$
\begin{aligned}
\left\|V\left(\rho_{1}\right)-V\left(\rho_{2}\right)\right\|_{\mathbf{L}^{\infty}(\mathbb{R} ; \mathbb{R})} & \leq\left\|v^{\prime}\right\|_{\mathbf{L}^{\infty}(\mathbb{R} ; \mathbb{R})}\left\|\rho_{1}-\rho_{2}\right\|_{\mathbf{L}^{1}(\mathbb{R} ; \mathbb{R})}, \\
\left\|\partial_{x} V\left(\rho_{1}\right)-\partial_{x} V\left(\rho_{2}\right)\right\|_{\mathbf{L}^{1}(\mathbb{R} ; \mathbb{R})} & =0,
\end{aligned}
$$

so that (V1) is satisfied. Similarly, $\partial_{x}^{2} V(\rho)=0$ and $\partial_{x}^{3} V(\rho)=0$ imply easily that (V2) and (V3) are satisfied.

We consider now (V4): is $v$ is $\mathcal{C}^{\mathbf{2}}$ then, for all $A, B \in \mathbb{R}$,

$$
v(B)=v(A)+v^{\prime}(A)(B-A)+\int_{0}^{1} v^{\prime \prime}(s B+(1-s) A)(1-s)(B-A)^{2} \mathrm{~d} s
$$

Choosing $A=\int_{0}^{1} \rho(\xi) \mathrm{d} \xi$ and $B=\int_{0}^{1} \tilde{\rho}(\xi) \mathrm{d} \xi$, we get

$$
\left\|v\left(\int_{0}^{1} \tilde{\rho}(\xi) \mathrm{d} \xi\right)-v\left(\int_{0}^{1} \rho(\xi) \mathrm{d} \xi\right)-v^{\prime}\left(\int_{0}^{1} \rho(\xi) \mathrm{d} \xi\right) \int_{0}^{1}(\tilde{\rho}-\rho)(\xi) \mathrm{d} \xi\right\|_{\mathbf{L}^{\infty}} \leq \frac{1}{2}\left\|v^{\prime \prime}\right\|_{\mathbf{L}^{\infty}}\|\tilde{\rho}-\rho\|_{\mathbf{L}^{1}}^{2}
$$

and we choose $K=\frac{1}{2}\left\|v^{\prime \prime}\right\|_{\mathbf{L}^{\infty}}, \mathrm{D} V(\rho)(r)=v^{\prime}\left(\int_{0}^{1} \rho(\xi) \mathrm{d} \xi\right) \int_{0}^{1} r(\xi) \mathrm{d} \xi$. Condition (V4) is then satisfied since there is no $x$-dependence, so

$$
\begin{aligned}
\|V(\tilde{\rho})-V(\rho)-\operatorname{D} V(\rho)(\tilde{\rho}-\rho)\|_{\mathbf{W}^{2, \infty}} & =\|V(\tilde{\rho})-V(\rho)-\mathrm{D} V(\rho)(\tilde{\rho}-\rho)\|_{\mathbf{L}^{\infty}} \\
& \leq \frac{1}{2}\left\|v^{\prime \prime}\right\|_{\mathbf{L}^{\infty}}\|\tilde{\rho}-\rho\|_{\mathbf{L}^{1}}^{2} .
\end{aligned}
$$

Similarly, $\|\mathrm{D} V(\rho)(r)\|_{\mathbf{W}^{2, \infty}}=\|\mathrm{D} V(\rho)(r)\|_{\mathbf{L}^{\infty}} \leq\left\|v^{\prime}\right\|_{\mathbf{L}^{\infty}}\|r\|_{\mathbf{L}^{1}}$. Finally, consider (V5):

$$
\begin{aligned}
& \left\|\operatorname{div}\left[v\left(\int_{0}^{1} \tilde{\rho}(\xi) \mathrm{d} \xi\right)-v\left(\int_{0}^{1} \rho(\xi) \mathrm{d} \xi\right)-v^{\prime}\left(\int_{0}^{1} \rho(\xi) \mathrm{d} \xi\right) \int_{0}^{1}(\tilde{\rho}-\rho)(\xi) \mathrm{d} \xi\right]\right\|_{\mathbf{L}^{1}}=0 \\
& \left\|\operatorname{div}\left(v^{\prime}\left(\int_{0}^{1} \rho(\xi) \mathrm{d} \xi\right) \int_{0}^{1} r(\xi) \mathrm{d} \xi\right)\right\|_{\mathbf{L}^{1}}=0 .
\end{aligned}
$$

Concluding the proof.

Proof of Proposition 4.1. The proof exploits the standard properties of the convolution. 
Consider first (V1):

$$
\begin{aligned}
\left\|\nabla_{x} V(\rho)\right\|_{\mathbf{L}^{\infty}} & =\left\|v^{\prime}\right\|_{\mathbf{L}^{\infty}}\|\rho\|_{\mathbf{L}^{\infty}}\left\|\nabla_{x} \eta\right\|_{\mathbf{L}^{1}}\|\vec{v}\|_{\mathbf{L}^{\infty}}+\|v\|_{\mathbf{L}^{\infty}}\left\|\nabla_{x} \vec{v}\right\|_{\mathbf{L}^{\infty}} \\
\leq & C\left(\|\rho\|_{\mathbf{L}^{\infty}}\right) \\
\left\|\nabla_{x} V(\rho)\right\|_{\mathbf{L}^{1}} \leq & \|v\|_{\mathbf{W}^{1, \infty}}\|\vec{v}\|_{\mathbf{W}^{1,1}}\left(1+\|\rho\|_{\mathbf{L}^{\infty}}\left\|\nabla_{x} \eta\right\|_{\mathbf{L}^{1}}\right), \\
\left\|\nabla_{x}^{2} V(\rho)\right\|_{\mathbf{L}^{1}} \leq & \|v\|_{\mathbf{W}^{2, \infty}}\|\vec{v}\|_{\mathbf{W}^{2,1}} \\
& \times\left[1+\|\rho\|_{\mathbf{L}^{\infty}}^{2}\left\|\nabla_{x} \eta\right\|_{\mathbf{L}^{1}}^{2}+\|\rho\|_{\mathbf{L}^{\infty}}\left\|\nabla_{x}^{2} \eta\right\|_{\mathbf{L}^{1}}+2\|\rho\|_{\mathbf{L}^{\infty}}\left\|\nabla_{x} \eta\right\|_{\mathbf{L}^{1}}\right] \\
\leq & C\left(\|\rho\|_{\mathbf{L}^{\infty}}\right), \\
\left\|V\left(\rho_{1}\right)-V\left(\rho_{2}\right)\right\|_{\mathbf{L}^{\infty}} \leq & \left\|v^{\prime}\right\|_{\mathbf{L}^{\infty}}\|\vec{v}\|_{\mathbf{L}^{\infty}}\|\eta\|_{\mathbf{L}^{\infty}}\left\|\rho_{1}-\rho_{2}\right\|_{\mathbf{L}^{1}}, \\
\left\|\nabla_{x}\left(V\left(\rho_{1}\right)-V\left(\rho_{2}\right)\right)\right\|_{\mathbf{L}^{1}}= & \|v\|_{\mathbf{W}^{2, \infty}}\|\vec{v}\|_{\mathbf{W}^{1, \infty}}\|\eta\|_{\mathbf{W}^{1,1}}\left(2+\left\|\nabla_{x} \eta\right\|_{\mathbf{L}^{1}}\left\|\rho_{1}\right\|_{\mathbf{L}^{\infty}}\right)\left\|\rho_{1}-\rho_{2}\right\|_{\mathbf{L}^{1}} .
\end{aligned}
$$

Then, we check (V2):

$$
\begin{aligned}
\left\|\nabla_{x}^{2} V(\rho)\right\|_{\mathbf{L}^{\infty}} \leq & 2\|v\|_{\mathbf{W}^{2, \infty}}\|\vec{v}\|_{\mathbf{W}^{2, \infty}} \\
& \times\left(1+\|\rho\|_{\mathbf{L}^{\infty}}^{2}\left\|\nabla_{x} \eta\right\|_{\mathbf{L}^{1}}^{2}+\|\rho\|_{\mathbf{L}^{\infty}}\left\|\nabla_{x}^{2} \eta\right\|_{\mathbf{L}^{1}}+\|\rho\|_{\mathbf{L}^{\infty}}\left\|\nabla_{x} \eta\right\|_{\mathbf{L}^{1}}\right) .
\end{aligned}
$$

Entirely analogous computations allow to prove also (V3).

Consider (V4). First we look at the Fréchet derivative of $V(\rho): v$ being $\mathcal{C}^{2}$, we can write, for all $A, B \in \mathbb{R}$,

$$
v(B)=v(A)+v^{\prime}(A)(B-A)+\int_{0}^{1} v^{\prime \prime}(s B+(1-s) A)(1-s)(B-A)^{2} \mathrm{~d} s .
$$

If we take $A=\rho * \eta$ and $B=\tilde{\rho} * \eta$, then we get, for $\rho, \tilde{\rho} \in \mathbf{L}^{\mathbf{1}}\left(\mathbb{R}^{N} ; \mathbb{R}\right)$

$$
\left\|\left(v(\tilde{\rho} * \eta)-v(\rho * \eta)-v^{\prime}(\rho * \eta)((\tilde{\rho}-\rho) * \eta)\right) \vec{v}\right\|_{\mathbf{L}^{\infty}} \leq \frac{1}{2}\left\|v^{\prime \prime}\right\|_{\mathbf{L}^{\infty}}\|\eta\|_{\mathbf{L}^{\infty}}^{2}\|\tilde{\rho}-\rho\|_{\mathbf{L}^{1}}^{2}\|\vec{v}\|_{\mathbf{L}^{\infty}} ;
$$

and

$$
\begin{aligned}
& \left\|\nabla_{x}\left[\left(v(\tilde{\rho} * \eta)-v(\rho * \eta)-v^{\prime}(\rho * \eta)((\tilde{\rho}-\rho) * \eta)\right) \vec{v}\right]\right\|_{\mathbf{L}^{\infty}} \leq \frac{3}{2}\left\|v^{\prime \prime}\right\|_{\mathbf{L}^{\infty}}\|\eta\|_{\mathbf{W}^{1, \infty}}^{2}\|\tilde{\rho}-\rho\|_{\mathbf{L}^{1}}^{2}\|\vec{v}\|_{\mathbf{W}^{1, \infty}} \\
& \quad+\frac{1}{2}\left\|v^{\prime \prime \prime}\right\|_{\mathbf{L}^{\infty}}\|\eta\|_{\mathbf{L}^{\infty}}^{2}\|\rho\|_{\mathbf{L}^{\infty}}\left\|\nabla_{x} \eta\right\|_{\mathbf{L}^{1}}\|\tilde{\rho}-\rho\|_{\mathbf{L}^{1}}^{2}\|\vec{v}\|_{\mathbf{L}^{\infty}} ; \\
& \| \nabla_{x}^{2}\left[\left(v(\tilde{\rho} * \eta)-v(\rho * \eta)-v^{\prime}(\rho * \eta)((\tilde{\rho}-\rho) * \eta)\right) \vec{v} \|_{\mathbf{L}^{\infty}}\right. \\
& \leq\left\|v^{(4)}\right\|_{\mathbf{L}^{\infty}}\|\tilde{\rho}-\rho\|_{\mathbf{L}^{1}}^{2}\|\eta\|_{\mathbf{L}^{\infty}}^{2}\left\|\nabla_{x} \eta\right\|_{\mathbf{L}^{1}}^{2}\left(\|\rho\|_{\mathbf{L}^{\infty}}+\|\tilde{\rho}\|_{\mathbf{L}^{\infty}}\right)^{2}\|\vec{v}\|_{\mathbf{L}^{\infty}} \\
& \quad+2\left\|v^{(3)}\right\|_{\mathbf{L}^{\infty}}\|\tilde{\rho}-\rho\|_{\mathbf{L}^{1}}^{2}\|\eta\|_{\mathbf{W}^{1, \infty}}^{2}\left(\|\rho\|_{\mathbf{L}^{\infty}}+\|\tilde{\rho}\|_{\mathbf{L}^{\infty}}\right)\|\nabla \eta\|_{\mathbf{L}^{1}}\|\vec{v}\|_{\mathbf{L}^{\infty}} \\
& \quad+\frac{1}{2}\left\|v^{(3)}\right\|_{\mathbf{L}^{\infty}}\|\tilde{\rho}-\rho\|_{\mathbf{L}^{1}}^{2}\|\eta\|_{\mathbf{L}^{\infty}}^{2}\left(\|\rho\|_{\mathbf{L}^{\infty}}\|\eta\|_{\mathbf{W}^{2,1}}+1\right)\|\vec{v}\|_{\mathbf{W}^{1, \infty}} \\
& \quad+6\left\|v^{\prime \prime}\right\|_{\mathbf{L}^{\infty}}\|\tilde{\rho}-\rho\|_{\mathbf{L}^{1}}^{2}\|\eta\|_{\mathbf{W}^{2, \infty}}^{2}\|\vec{v}\|_{\mathbf{W}^{2, \infty}} .
\end{aligned}
$$


Then, $\mathrm{D} V(\rho)(r)=v^{\prime}(\rho * \eta) r * \eta \vec{v}$. To satisfy (V4), we have to check that the derivative is a bounded operator from $\mathcal{C}^{2}$ to $\mathbf{L}^{1}$. We have,

$$
\begin{aligned}
\|\mathrm{D} V(\rho)(r)\|_{\mathbf{L}^{\infty}} \leq & \left\|v^{\prime}\right\|_{\mathbf{L}^{\infty}}\|\eta\|_{\mathbf{L}^{\infty}}\|\vec{v}\|_{\mathbf{L}^{\infty}}\|r\|_{\mathbf{L}^{1}} \\
\left\|\nabla_{x} \mathrm{D} V(\rho)(r)\right\|_{\mathbf{L}^{\infty}} \leq & \|v\|_{\mathbf{W}^{2, \infty}}\|\vec{v}\|_{\mathbf{W}^{1, \infty}}\|\eta\|_{\mathbf{W}^{1, \infty}}\left(2+\|\rho\|_{\mathbf{L}^{\infty}}\|\eta\|_{\mathbf{W}^{1,1}}\right)\|r\|_{\mathbf{L}^{1}} \\
\left\|\nabla_{x}^{2} \mathrm{D} V(\rho)(r)\right\|_{\mathbf{L}^{\infty}} \leq & \|v\|_{\mathbf{W}^{3, \infty}}\|\eta\|_{\mathbf{W}^{2, \infty}}\|\vec{v}\|_{\mathbf{W}^{2, \infty}} \\
& \times\left(4+5\|\rho\|_{\mathbf{L}^{\infty}}\|\eta\|_{\mathbf{W}^{2,1}}+\|\rho\|_{\mathbf{L}^{\infty}}^{2}\|\nabla \eta\|_{\mathbf{L}^{1}}^{2}\right)\|r\|_{\mathbf{L}^{1}} .
\end{aligned}
$$

Finally, we check that also (V5) is satisfied:

$$
\begin{aligned}
& \|\operatorname{div}(V(\tilde{\rho})-V(\rho)-\operatorname{D} V(\rho)(\tilde{\rho}-\rho))\|_{\mathbf{L}^{1}} \\
\leq & \frac{1}{2}\|v\|_{\mathbf{W}^{3, \infty}}\|\tilde{\rho}-\rho\|_{\mathbf{L}^{1}}^{2}\|\eta\|_{\mathbf{L}^{1}}\|\eta\|_{\mathbf{W}^{1, \infty}}\|\vec{v}\|_{\mathbf{W}^{1, \infty}}\left(3+\|\rho\|_{\mathbf{L}^{\infty}}\|\eta\|_{\mathbf{W}^{1,1}}\right) \\
& \|\operatorname{div} \operatorname{DV}(\rho)(r)\|_{\mathbf{L}^{1}} \\
= & \left\|\operatorname{div}\left(v^{\prime}(\rho * \eta) r * \eta \vec{v}\right)\right\|_{\mathbf{L}^{1}} \\
\leq & \|v\|_{\mathbf{W}^{2, \infty}}\|\eta\|_{\mathbf{W}^{1,1}}\|\vec{v}\|_{\mathbf{W}^{1, \infty}}\left(2+\|\rho\|_{\mathbf{L}^{\infty}}\left\|\nabla_{x} \eta\right\|_{\mathbf{L}^{1}}\right)\|r\|_{\mathbf{L}^{1}}
\end{aligned}
$$

completing the proof.

Remark 5.5. The above proof shows that (B) is not satisfied by (4.1): here $C$ grows linearly, $C(\alpha)=1+\alpha$. Hence, with the notation in the proof of Theorem 2.4, for $\alpha_{1}>0$,

$$
\sum_{k=1}^{n} T\left(\alpha_{k}, \alpha_{k+1}\right) \leq \sum_{k=1}^{n} \frac{1}{1+\alpha_{k+1}} \int_{\alpha_{k}}^{\alpha_{k+1}} \frac{1}{t} \mathrm{~d} t \leq \sum_{k=1}^{n} \int_{\alpha_{k}}^{\alpha_{k+1}} \frac{1}{(1+t) t} \mathrm{~d} t \leq \int_{\alpha_{1}}^{+\infty} \frac{1}{(1+t) t} \mathrm{~d} t
$$

and the latter expression is bounded. This shows that, in the case of (4.1), the technique used in Theorem 2.4 does not apply.

Acknowledgements. The authors are indebted with a referee whose remarks allowed to simplify the proof of Lemma 5.1 and to overall significantly improve the paper. This work has been supported by the DFG SPP1253, DAAD D/06/19582, DAAD D/08/11076, and HE5386/6-1. The third author thanks Sylvie Benzoni-Gavage for useful discussions.

\section{REFERENCES}

[1] C.E. Agnew, Dynamic modeling and control of congestion-prone systems. Oper. Res. 24 (1976) 400-419.

[2] L. Ambrosio, Transport equation and Cauchy problem for non-smooth vector fields, in Calculus of variations and nonlinear partial differential equations, Lecture Notes in Math. 1927, Springer, Berlin, Germany (2008) 1-41.

[3] D. Armbruster, P. Degond and C. Ringhofer, A model for the dynamics of large queuing networks and supply chains. SIAM J. Appl. Math. 66 (2006) 896-920.

[4] D. Armbruster, D.E. Marthaler, C. Ringhofer, K. Kempf and T.-C. Jo, A continuum model for a re-entrant factory. Oper. Res. 54 (2006) 933-950.

[5] S. Benzoni-Gavage, R.M. Colombo and P. Gwiazda, Measure valued solutions to conservation laws motivated by traffic modelling. Proc. R. Soc. Lond. Ser. A Math. Phys. Eng. Sci. 462 (2006) 1791-1803.

[6] S. Bianchini, On the shift differentiability of the flow generated by a hyperbolic system of conservation laws. Discrete Contin. Dynam. Systems 6 (2000) 329-350.

[7] F. Bouchut and F. James, One-dimensional transport equations with discontinuous coefficients. Nonlinear Anal. 32 (1998) 891-933.

[8] F. Bouchut and F. James, Differentiability with respect to initial data for a scalar conservation law, in Hyperbolic problems: theory, numerics, applications, Internat. Ser. Numer. Math., Birkhäuser, Basel, Switzerland (1999).

[9] A. Bressan and G. Guerra, Shift-differentiability of the flow generated by a conservation law. Discrete Contin. Dynam. Systems 3 (1997) 35-58. 
[10] A. Bressan and M. Lewicka, Shift differentials of maps in BV spaces, in Nonlinear theory of generalized functions (Vienna, 1997), Res. Notes Math. 401, Chapman \& Hall/CRC, Boca Raton, USA (1999) 47-61.

[11] A. Bressan and W. Shen, Optimality conditions for solutions to hyperbolic balance laws, in Control methods in PDE-dynamical systems, Contemp. Math. 426, AMS, USA (2007) 129-152.

[12] C. Canuto, F. Fagnani and P. Tilli, A eulerian approach to the analysis of rendez-vous algorithms, in Proceedings of the IFAC World Congress (2008)

[13] R.M. Colombo and A. Groli, On the optimization of the initial boundary value problem for a conservation law. J. Math. Analysis Appl. 291 (2004) 82-99.

[14] R.M. Colombo and M.D. Rosini, Pedestrian flows and non-classical shocks. Math. Methods Appl. Sci. 28 (2005) $1553-1567$.

[15] R.M. Colombo, M. Mercier and M.D. Rosini, Stability and total variation estimates on general scalar balance laws. Commun. Math. Sci. 7 (2009) 37-65.

[16] R.M. Colombo, G. Facchi, G. Maternini and M.D. Rosini, On the continuum modeling of crowds, in Hyperbolic Problems: Theory, Numerics, Applications 67, Proceedings of Symposia in Applied Mathematics, E. Tadmor, J.-G. Liu and A.E. Tzavaras Eds., American Mathematical Society, Providence, USA (2009).

[17] V. Coscia and C. Canavesio, First-order macroscopic modelling of human crowd dynamics. Math. Models Methods Appl. Sci. 18 (2008) 1217-1247.

[18] M. Gugat, M. Herty, A. Klar and G. Leugering, Conservation law constrained optimization based upon Front-Tracking. ESAIM: M2AN 40 (2006) 939-960.

[19] R.L. Hughes, A continuum theory for the flow of pedestrians. Transportation Res. Part B 36 (2002) 507-535.

[20] U. Karmarkar, Capacity loading and release planning in work-in-progess (wip) and lead-times. J. Mfg. Oper. Mgt. 2 (1989) $105-123$.

[21] S.N. Kružkov, First order quasilinear equations with several independent variables. Mat. Sb. (N.S.) 81 (1970) $228-255$.

[22] M. Marca, D. Armbruster, M. Herty and C. Ringhofer, Control of continuum models of production systems. IEEE Trans. Automat. Contr. (to appear).

[23] B. Perthame and A.-L. Dalibard, Existence of solutions of the hyperbolic Keller-Segel model. Trans. Amer. Math. Soc. 361 (2009) 2319-2335.

[24] S. Ulbrich, A sensitivity and adjoint calculus for discontinuous solutions of hyperbolic conservation laws with source terms. SIAM J. Control Optim. 41 (2002) 740.

[25] S. Ulbrich, Adjoint-based derivative computations for the optimal control of discontinuous solutions of hyperbolic conservation laws. Syst. Contr. Lett. 48 (2003) 313-328.

[26] V.I. Yudovič, Non-stationary flows of an ideal incompressible fluid. Z̆. Vyčisl. Mat. i Mat. Fiz. 3 (1963) 1032-1066. 